\title{
A nonlinear integral transform and a global inverse bifurcation theory
}

\author{
YUTAKA KAMIMURA
}

\begin{abstract}
We consider a nonlinear integral transform and show that the transform acts as a homeomorphism between certain metric spaces of positive functions. We apply the result to the inverse bifurcation problem of determining the nonlinear term of a certain nonlinear Sturm-Liouville problem from its first bifurcating branch, and we establish the well-posedness of the inverse problem. An application to an inverse problem of determining a restoring force from a timemap is also given.
\end{abstract}

Mathematics Subject Classification (2010): 44A15 (primary); 34A55, 45P05 (secondary).

\section{The main result}

This paper studies the nonlinear integral transform $\mathcal{K}$ defined by

$$
(\mathcal{K} f)(x)=\int_{0}^{1} \frac{d t}{\left(\int_{t}^{1} s^{\beta-1} f(x s) d s\right)^{1-\delta}}, \quad x \in I,
$$

where $0<\delta<1, \beta>0$, and $I$ is a bounded, closed interval containing 0 . Our objective is to show that the transform $\mathcal{K}$ is a homeomorphism of an appropriate metric space onto a twin metric space reflecting the smoothing property of $\mathcal{K}$ and, as its application, that an inverse problem to determine a nonlinear term of a certain nonlinear Sturm-Liouville problem from its first bifurcating branch is globally wellposed.

Throughout the paper, $\theta$ denotes the Euler differential operator $\theta=x \frac{d}{d x}$. To state the main result explicitly, for a nonnegative integer $k$, a number $\alpha \in(0,1]$,

Supported by Grant-in-Aid for Scientific Research No. 21540165, Japan Society for Promotion of Science.

Received February 12, 2009; accepted in revised form July 29, 2010. 
and a number $\eta \in \mathbb{R}$, we introduce the function space

$$
\mathcal{C}^{k, \alpha}(I)_{\eta}:=\left\{\phi \in C^{k}(I \backslash\{0\}):\|\phi\|_{k, \alpha, \eta}=\sum_{i=0}^{k}\left|\theta^{i} \phi\right|_{\eta}+\left|\theta^{k} \phi\right|_{\alpha, \eta}<\infty\right\}
$$

where $|\cdot|_{\eta}$ and $|\cdot|_{\alpha, \eta}$ are the semi-norms defined by

$$
|\phi|_{\eta}:=\sup _{x \in I \backslash\{0\}} \frac{|\phi(x)|}{|x|^{\eta}}, \quad|\phi|_{\alpha, \eta}:=\sup _{x, y \in I \backslash\{0\}, x \neq y} \frac{\left.|| x\right|^{\alpha-\eta} \phi(x)-|y|^{\alpha-\eta} \phi(y) \mid}{|x-y|^{\alpha}} .
$$

Equipped with the norm $\|\phi\|_{k, \alpha, \eta}$, the space $\mathcal{C}^{k, \alpha}(I)_{\eta}$ is a Banach space. A suitable choice of metric spaces on which $\mathcal{K}$ acts is $\mathcal{M}^{k, \alpha}(I)_{\eta}$ defined by

$$
\mathcal{M}^{k, \alpha}(I)_{\eta}:=\left\{f \in C_{+}(I): f(x)-f(0) \in \mathcal{C}^{k, \alpha}(I)_{\eta}\right\}
$$

with the metric

$$
d(f, g):=|f(0)-g(0)|+\|(f(x)-f(0))-(g(x)-g(0))\|_{k, \alpha, \eta} .
$$

Here $C_{+}(I)$ denotes the set of positive, continuous functions on $I$ :

$$
C_{+}(I):=\{f \in C(I): f(x)>0 \text { for any } x \in I\} .
$$

One of the main theorems in this paper is now stated as follows:

Theorem 1.1. Let $0<\delta<1, \beta>0$, and let I be a bounded, closed interval containing 0 . Then, for any $\alpha, \eta$ such that $0<\eta \leq \alpha<1-\delta$, the transform $\mathcal{K}$ is a homeomorphism of $\mathcal{M}^{1, \alpha}(I)_{\eta}$ onto $\mathcal{M}^{1, \alpha+\delta}(I)_{\eta}$.

The assertion in Theorem 1.1 is the same as saying that, given $F \in \mathcal{M}^{1, \alpha+\delta}(I)_{\eta}$, the nonlinear integral equation

$$
\int_{0}^{1} \frac{d t}{\left(\int_{t}^{1} s^{\beta-1} f(x s) d s\right)^{1-\delta}}=F(x), \quad x \in I,
$$

has a unique solution $f$ in $\mathcal{M}^{1, \alpha}(I)_{\eta}$ and both correspondences $f \mapsto F$ and $F \mapsto$ $f$ are continuous in the sense of the metrics of the two spaces involved. The proof of Theorem 1.1 is organized as follows. In Section 2 we shall prove that equation (1.5) has a local solution near $x=0$ by using an implicit function theorem on function spaces and a theory of multiplicative Wiener-Hopf equations developed by Iwasaki-Kamimura $[12,13]$. In recasting neighborhoods of 0 in the function spaces to an interval $I_{\kappa}:=\{\kappa x: x \in I\}$ near $x=0$, we employ a scaling operator $S_{\kappa}$ defined by $\left(S_{\kappa} f\right)(x)=f(\kappa x)$. The commutative property $\mathcal{K} S_{\kappa}=S_{\kappa} \mathcal{K}$ of the transform $\mathcal{K}$ on the scaling operator makes it possible to obtain a solution of 
the equation on the interval $I_{\kappa}$ with sufficiently small $\kappa$. In Section 3 we explore a significant characteristic of the equation; a solution $f$ of the equation remains in the space $\mathcal{M}^{1, \alpha}(I)_{\eta}$ as long as $F$ belongs to $\mathcal{M}^{1, \alpha+\delta}(I)_{\eta}$. To show this good behavior of the solution we make use of the so-called fractional calculus together with a technique established in Kamimura [17]. Loosely speaking, by differentiating (1.5) $1-\varepsilon$ times and then letting $\varepsilon$ tend to 0 , we shall prove that the solution never goes to infinity. An explicit statement and a rigorous proof of this fact is given in Lemma 3.9. The characteristic of the equation is rather delicate. Actually a solution of (1.5) does not necessarily remain in the space $\mathcal{M}^{0, \alpha}(I)_{\eta}$ even though $F$ belongs to $\mathcal{M}^{0, \alpha+\delta}(I)_{\eta}$, as will be indicated by Example 4.5 at the end of Section 4; the conclusion in Theorem 1.1 breaks down generally when a pair $\mathcal{M}^{1, \alpha}(I)_{\eta}$ and $\mathcal{M}^{1, \alpha+\delta}(I)_{\eta}$ is replaced by $\mathcal{M}^{0, \alpha}(I)_{\eta}$ and $\mathcal{M}^{0, \alpha+\delta}(I)_{\eta}$. For this reason the proof of Lemma 3.9 becomes considerably technical. In Section 4 we shall prove that each solution $f \in \mathcal{M}^{1, \alpha}\left(I^{\prime}\right)_{\eta}$ of the equation on a closed subinterval $I^{\prime} \subset I$ can be extended to both sides of $I^{\prime}$. Following this observation, in Proposition 4.3 we shall prove that our transform $\mathcal{K}$ is a bijection from $\mathcal{M}^{1, \alpha}(I)_{\eta}$ onto $\mathcal{M}^{1, \alpha+\delta}(I)_{\eta}$. The continuities of the correspondences $f \mapsto F$ and $F \mapsto f$ are established in Lemma 2.3 and Proposition 5.4, respectively.

Our study on the transform $\mathcal{K}$ is motivated by an inverse problem in bifurcation theory (inverse bifurcation problem). Let $f$ be a positive, continuous function on a bounded, closed interval $I$ containing 0 and consider the nonlinear eigenvalue problem

$$
\left\{\begin{array}{l}
u^{\prime \prime}+\lambda u f(u)=0 \text { on }(0,1),{ }^{\prime}=\frac{d}{d x} \\
u(0)=u(1)=0 \\
u \neq 0 \text { on }(0,1)
\end{array}\right.
$$

By the condition that $u \neq 0$ on the interval $(0,1)$, each solution $u$ of (1.6) is positive or negative in the interval. Hence the solution has its maximum or minimum value at the middle point $\frac{1}{2}$ of the interval. By means of the value $h$, as a projection into $(0, \infty) \times I$ of the first bifurcating branch of (1.6), we define a set $\Gamma(f)$ in $(0, \infty) \times I$ by

$$
\Gamma(f):=\left\{(\lambda, h) \in(0, \infty) \times I \mid \exists u \in C^{2}[0,1] \text { satisfying (1.6) and } u\left(\frac{1}{2}\right)=h\right\} .
$$

Then, as is easily seen (see Lemma 6.1 in Section 6), the set $\Gamma(f)$ is expressed as $\Gamma(f)=\{(\lambda(h), h): h \in I \backslash\{0\}\}$ in terms of a positive function $\lambda(h)$ defined by

$$
\lambda(h)=2\left(\int_{0}^{1} \frac{d t}{\sqrt{\int_{t}^{1} s f(h s) d s}}\right)^{2} .
$$

We thus have a correspondence (the forward bifurcation transform)

$$
\mathcal{B}: f(u) \mapsto \lambda(h)
$$




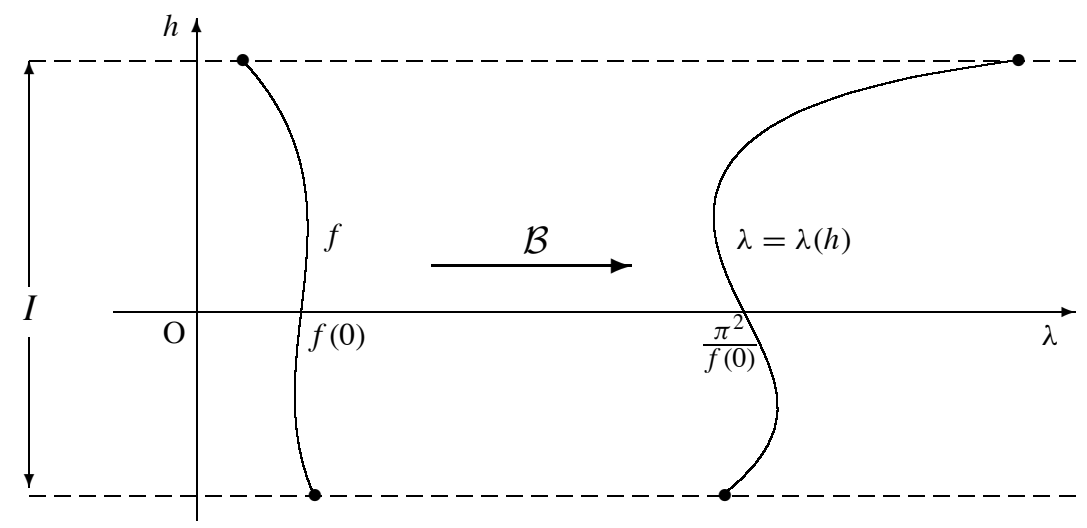

Figure 1.1. The bifurcation transform.

(see Figure 1.1), where the trivial function $f \equiv f(0)$ corresponds to the trivial bifurcation $\lambda(h) \equiv \frac{\pi^{2}}{f(0)}$ in the linear case.

The inverse bifurcation problem is to ask whether $f$ can be recovered from $\lambda(h)$. By (1.8), the bifurcation transform $\mathcal{B}$ is written as $\mathcal{B} f=2(\mathcal{K} f)^{2}$ in terms of the transform $\mathcal{K}$ with $\delta=\frac{1}{2}, \beta=2$ in (1.1). Accordingly we can draw the following conclusion from Theorem 1.1 .

Theorem 1.2. For any $\alpha$, $\eta$ such that $0<\eta \leq \alpha<\frac{1}{2}$, the bifurcation transform $\mathcal{B}$ is a homeomorphism of $\mathcal{M}^{1, \alpha}(I)_{\eta}$ onto $\mathcal{M}^{1, \alpha+\frac{1}{2}}(I)_{\eta}$.

The rigorous statement of the inverse bifurcation problem and an example indicating the meaning of Theorem 1.2 is given in Section 6.

The inverse problem to determine a nonlinear term from a first bifurcating branch was studied in Kamimura [14], Iwasaki and Kamimura [11,13] for a class of Sturm-Liouville equations different from (1.6). In these papers, the local existence of nonlinear terms realizing a prescribed bifurcating branch was established. That is, it was proved that, for each curve sufficiently near the trivial bifurcation in the linear case, there exist nonlinear terms (not unique, in general) realizing the curve as their first bifurcating branch. Studies for some related inverse problems to determine nonlinear terms of semilinear differential equations from spectral data can be found in Zhidkov [33], Shibata [29]. Theorem 1.2 asserts that the inverse bifurcation problem is well-posed globally for the simpler problem (1.6).

The inverse bifurcation problem mentioned above is directly connected to a classical inverse problem (inverse time-map problem): determine a function $g$ representing a restoring force in the newtonian equation $\ddot{u}+g(u)=0$ from a time-map $T(h)$ assigning to each half-amplitude the corresponding half-period. In fact, by a 
scaling $\sqrt{\lambda} x=t$ and the setting $g(u)=u f(u)$, the problem (1.6) becomes

$$
\left\{\begin{array}{l}
\ddot{u}+g(u)=0 \text { on }(0, \sqrt{\lambda}), \cdot=\frac{d}{d t}, \\
u(0)=u(\sqrt{\lambda})=0 \\
u>0 \text { on }(0, \sqrt{\lambda})
\end{array}\right.
$$

provided that $I=[0, b]$ and $\lambda>0$. Hence the mapping $T: h \mapsto \sqrt{\lambda}$ is no other than the time-map (see Figure 1.2). In view of (1.8), the time-map associated to $g$ is expressed as

$$
T(h)=\sqrt{2} \int_{0}^{1} \frac{d t}{\sqrt{\int_{t}^{1} s f(h s) d s}}=\sqrt{2} \int_{0}^{h} \frac{d \eta}{\sqrt{\int_{\eta}^{h} g(\xi) d \xi}}
$$

or written as

$$
T=\sqrt{2} \mathcal{K} f
$$

in terms of the transform $\mathcal{K}$ with $\delta=\frac{1}{2}, \beta=2$ in (1.1).

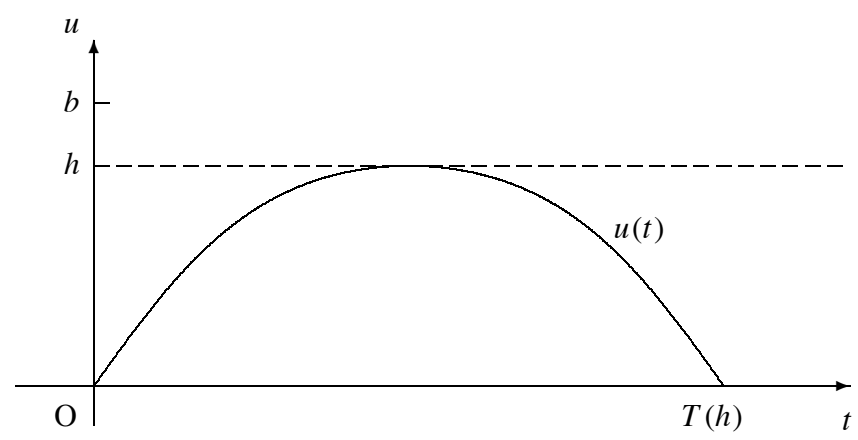

Figure 1.2. The time-map.

The inverse time-map problem has been studied in many publications. The first important contribution to the problem was given in Opial [23], which showed that if two time-maps $T_{i}(h)$ associated to continuous functions $g_{i}, i=1,2$, satisfy $T_{1}(h) \leq T_{2}(h)$ for all $h>0$ then $\int_{0}^{h} g_{1}(\xi) d \xi \geq \int_{0}^{h} g_{2}(\xi) d \xi$ for all $h \geq 0$. The uniqueness of a continuous $g$ for each given $T$ is immediate from this result. The local existence of $g$ was first established in Urabe [30,31] (see also [32]) under the assumption that the derivative of $T$ is Lipschitz continuous. This local existence result was improved in Alfawicka [1, Theorem 3.2], which proved the local existence of $g$ under the assumption that $T$ itself is Lipschitz continuous. The global version of Alfawicka's result has recently been established in Kamimura [18]:

Theorem 1.3 (18, Theorem 1.2 and equation (4.2)). Given a positive, Lipschitz continuous function $T$ on the interval $[0, b]$, there exists a (unique) continuous 
function $g$ defined on the interval $[0, b]$, positive on the interval $(0, b]$ and such that the time-map associated to g equals $T(h)$ for $h \in[0, b]$. Moreover,

$$
g(h)=(\pi / T(0))^{2} h+O\left(h^{2}\right), \quad \text { as } h \rightarrow 0 .
$$

It should be noticed that Alfawicka [2], Mañosas and Torres [22] studied the inverse time-map problem in a framework of analytic functions. Also some related topics in view of Hamiltonian systems may be found in Cima, Mañosas and Villadelprat [4], Cima, Gasull and Mañosas [3]. A similar inverse problem concerning a time-map assigning half-period (or period) to each energy level, in place of to each halfamplitude, has been studied in detail by Schaaf [27]. This inverse problem, which has been referred to as determination of the potential energy from the period of oscillations, can be reduced to Abel's integral equation (see Landau and Lifshitz [20, Section 12], Keller [19], Gorenflo and Vessella [9, Section 2.4]). In [27] this problem was discussed in connection with bifurcation theory. Also, in Henrard and Zanolin [10], it was discussed in an application to Hamiltonian systems. A class of inverse problem of determining $g$ of $u^{\prime \prime}=\lambda g(u)$ from a sort of time-map has also been considered in Denisov [6], Denisov and Lorenzi [7], Lorenzi [21], Kamimura [16] (see also Denisov and Lorenzi [8]).

Theorem 1.3 presents a global existence result to the inverse time-map problem. In addition, since the method in its proof is constructive, we can get a general strategy for reconstruction of $g$ (see [18, Corollary 4.3]). However Theorem 1.3 mentions neither bijectivity, namely whether the correspondence $g \mapsto T$ becomes a bijection between any suitable function spaces nor stability, namely whether $g$ depends continuously on $T$ in any reasonable topology. By applying Theorem 1.1 to the transform (1.12) we can give answers to these questions. For example, taking $\eta=\alpha$, we get the following answer:

Theorem 1.4. Let $\alpha$ be fixed in $(0,1 / 2)$. Then:

(1) A function $T(h)$ is the time-map associated to a function $g$ in the space

$$
X_{\alpha}:=\left\{g \in C^{1, \alpha}[0, b]: g(0)=0, g^{\prime}(0)>0, g(h)>0 \text { for any } h \in(0, b]\right\}
$$

if and only if $T(h)$ belongs to the space

$$
Y_{\alpha}:=\left\{T \in C_{+}[0, b] \cap C^{1}(0, b]: \lim _{h \rightarrow 0} h T^{\prime}(h)=0, \quad\left|h T^{\prime}(h)\right|_{\alpha+(1 / 2), \alpha}<\infty\right\} .
$$

Here $C^{1, \alpha}[0, b]$ denotes the standard Hölder space with exponent $1+\alpha$ and $|\cdot|_{\alpha+(1 / 2), \alpha}$ denotes a semi-norm defined by

$$
|\phi|_{\alpha+(1 / 2), \alpha}:=\sup _{0 \leq h \neq k \leq b} \frac{\left|h^{1 / 2} \phi(h)-k^{1 / 2} \phi(k)\right|}{|h-k|^{\alpha+(1 / 2)}} .
$$


(2) The correspondence $g \mapsto T$ is a continuous, one-to-one map from $X_{\alpha}$ onto $Y_{\alpha}$ and its inverse is also continuous with respect to the metrics

$$
\begin{aligned}
& d_{X}\left(g_{1}, g_{2}\right):=\left\|g_{1}^{\prime}-g_{2}^{\prime}\right\|_{\alpha}, \\
& d_{Y}\left(T_{1}, T_{2}\right):=\left|T_{1}(0)-T_{2}(0)\right|+\left|h\left(T_{1}-T_{2}\right)^{\prime}(h)\right|_{\alpha+(1 / 2), \alpha},
\end{aligned}
$$

where $\|\cdot\|_{\alpha}$ denotes a norm of $C^{0, \alpha}[0, b]$ :

$$
\|\phi\|_{\alpha}:=\sup _{0 \leq h \leq b}|\phi(h)|+\sup _{0 \leq h \neq k \leq b} \frac{|\phi(h)-\phi(k)|}{|h-k|^{\alpha}} .
$$

Roughly speaking, the metric space $Y_{\alpha}$ is of all positive functions such that the Euler derivative $h T^{\prime}(h)$ being Hölder continuous with exponent $\alpha+(1 / 2)$ at $h \neq 0$ and with exponent $\alpha$ at $h=0$, like $T(h)=T(0)+O\left(h^{\alpha}\right)$ as $h \rightarrow 0$.

Theorem 1.4 contains the following global existence result:

Corollary 1.5. Given a function $T \in Y_{\alpha}$, there exists a restoring force $g$ in $X_{\alpha}$ such that the time-map associated to $g$ equals $T$.

Since a typical function $g(h)=c h+h^{\alpha+1}$ in $X_{\alpha}$ with some $c>0, \alpha \in(0,1 / 2)$ does not satisfy (1.13), and so, the time-map associated to this function $g$ is not Lipschitz continuous. Thus it turns out that Theorems 1.3 and 1.4 are independent of each other.

In our framework, the inverse bifurcation problem is equivalent to the inverse time-map problem. The latter gives a vital, physical viewpoint to the former, while the former gives a wider perspective, at least, in three directions: Firstly it has a meaning even if $\lambda$ is non-positive though the time-map has no physical sense. Secondly, in ordinary differential equations, it can be posed even for the $n$th branch bifurcating at the $n$th eigenvalue from trivial solutions. In fact the case $n=2$ was treated in Kamimura [15] for a different class of equation from (1.6). Thirdly it can be formulated for a multi-dimensional equation, for example, $\Delta u+\lambda g(u)=0$ in an $n$-ball.

ACKNOWLEDGEMENTS. The author is very grateful to the referee for his valuable suggestions that improved the original manuscript.

\section{Local solvability}

In this section we shall develop a local theory. Let $I$ be a bounded, closed interval containing 0 and set $I_{\kappa}:=\{\kappa x: x \in I\}$ for $\kappa>0$. Then our goal in this section can be stated as follows:

Proposition 2.1. Let $0<\delta<1, \beta>0$, let $0<\eta \leq \alpha<1-\delta$, and let $F \in \mathcal{M}^{1, \alpha+\delta}(I)_{\eta}$. Then:

(1) If $\kappa>0$ is sufficiently small then the equation $\mathcal{K} f=F$ on $I_{\kappa}$ has a solution in $\mathcal{M}^{1, \alpha}\left(I_{K}\right)_{\eta}$. 
(2) If $f_{1}, f_{2}$ are solutions in $\mathcal{M}^{1, \alpha}(I)_{\eta}$ of $\mathcal{K} f=F$ on I then $f_{1}=f_{2}$ on $I_{\kappa}$ for sufficiently small $\kappa>0$.

Proposition 2.1 is proved by means of the implicit function and a scaling operator. Setting of a map and function spaces will be given as Lemmas 2.2 and 2.4 below. The proof of Proposition 2.1 will be given after Lemma 2.5.

To solve $\mathcal{K} f=F$, we may assume that $F(0)$ equals to

$$
\gamma:=\int_{0}^{1} \frac{d t}{\left(\int_{t}^{1} s^{\beta-1} d s\right)^{1-\delta}}
$$

by redefining $\gamma F(x) / F(0)$ so that we call $F(x)$. Noting that a solution $f$ of $\mathcal{K} f=$ $F$ for $F$ with $F(0)=\gamma$ satisfies $f(0)=1$, we define a transform $\mathcal{L}$ by

$$
(\mathcal{L} g)(x)=\int_{0}^{1} \frac{d t}{\left(\int_{t}^{1} s^{\beta-1}(1+g(x s)) d s\right)^{1-\delta}}-\int_{0}^{1} \frac{d t}{\left(\int_{t}^{1} s^{\beta-1} d s\right)^{1-\delta}}, \quad x \in I .
$$

Then the equation $\mathcal{K} f=F$ has a solution $f \in \mathcal{M}^{1, \alpha}(I)_{\eta}$ for $F \in \mathcal{M}^{1, \alpha+\delta}(I)_{\eta}$ if and only if $\mathcal{L}(f-1)=F-\gamma$ has a solution $f-1 \in \mathcal{C}^{1, \alpha}(I)_{\eta}$ for $F-\gamma \in$ $\mathcal{C}^{1, \alpha+\delta}(I)_{\eta}$.

We must solve $\mathcal{L} g=G$ in $\mathcal{C}^{1, \alpha}(I)_{\eta}$ for $G \in \mathcal{C}^{1, \alpha+\delta}(I)_{\eta}$ given in a neighborhood of 0 . We use the (local) implicit function theorem in the following framework.

Lemma 2.2. Let $0<\alpha<\alpha+\delta<1, \beta>0$, let $0 \leq \eta \leq \alpha$, and set

$$
U:=\left\{g \in \mathcal{C}^{1, \alpha}(I)_{\eta}: 1+g(x)>0 \text { for } x \in I\right\} .
$$

Then $\mathcal{L}$ defined by (2.2) is a $C^{1}$-map of $U$ to $\mathcal{C}^{1, \alpha+\delta}(I)_{\eta}$. The Fréchet derivative $\mathcal{L}^{\prime}\left(g_{0}\right)$ of $\mathcal{L}$ at $g_{0} \in U$ is given by

$$
\left(\mathcal{L}^{\prime}\left(g_{0}\right) g\right)(x)=(\delta-1) \int_{0}^{1} t^{\beta-1} g(x t) d t \int_{0}^{t} \frac{d r}{\left(\int_{r}^{1} s^{\beta-1}\left(1+g_{0}(x s)\right) d s\right)^{2-\delta}} .
$$

We omit the proof of Lemma 2.2, since it is standard. As a consequence of Lemma 2.2, we obtain:

Lemma 2.3. Let $0<\alpha<\alpha+\delta<1, \beta>0,0 \leq \eta \leq \alpha$. Then $\mathcal{K}$ defined by (1.1) is a continuous map from $\mathcal{M}^{1, \alpha}(I)_{\eta}$ to $\mathcal{M}^{1, \alpha+\delta}(I)_{\eta}$.

Proof. Define metric subspaces $\mathcal{M}_{0}^{1, \alpha}(I)_{\eta} \subset \mathcal{M}^{1, \alpha}(I)_{\eta}$ and $\mathcal{M}_{0}^{1, \alpha+\delta}(I)_{\eta} \subset \mathcal{M}^{1, \alpha+\delta}(I)_{\eta}$ by

$$
\begin{aligned}
& \mathcal{M}_{0}^{1, \alpha}(I)_{\eta}:=\left\{f \in \mathcal{M}^{1, \alpha}(I)_{\eta}: f(0)=1\right\}, \\
& \mathcal{M}_{0}^{1, \alpha+\delta}(I)_{\eta}:=\left\{F \in \mathcal{M}^{1, \alpha+\delta}(I)_{\eta}: F(0)=\gamma\right\} .
\end{aligned}
$$


Moreover, let $\omega_{\alpha}$ be a map that assigns $\left(\frac{f(x)}{f(0)}, f(0)\right)$ to each $f \in \mathcal{M}^{1, \alpha}(I)_{\eta}$. It is easy to see that $\omega_{\alpha}$ is a homeomorphism of $\mathcal{M}^{1, \alpha}(I)_{\eta}$ onto the product metric space $\mathcal{M}_{0}^{1, \alpha}(I)_{\eta} \times \mathbb{R}^{+}$, where $\mathbb{R}^{+}:=(0, \infty)$ is viewed as a metric space with a usual metric $|b-c|$ for $b, c \in \mathbb{R}^{+}$. Similarly the map $\omega_{\alpha+\delta}: F(x) \mapsto\left(\frac{\gamma}{F(0)} F(x), F(0)\right)$ is a homeomorphism of $\mathcal{M}^{1, \alpha+\delta}(I)_{\eta}$ onto the product metric space $\mathcal{M}_{0}^{1, \alpha+\delta}(I)_{\eta} \times$ $\mathbb{R}^{+}$. Furthermore we define a transform $\mathcal{K}_{0}$ from $\mathcal{M}_{0}^{1, \alpha}(I)_{\eta} \times \mathbb{R}^{+}$to $\mathcal{M}_{0}^{1, \alpha+\delta}(I)_{\eta} \times$ $\mathbb{R}^{+}$by $\mathcal{K}_{0}\left(f_{0}, c\right):=\left(\mathcal{K} f_{0}, \gamma / c^{1-\delta}\right)$. Then we have the following commutative diagram:

$$
\begin{array}{ccc}
\mathcal{M}^{1, \alpha}(I)_{\eta} & \stackrel{\mathcal{K}}{\longrightarrow} & \mathcal{M}^{1, \alpha+\delta}(I)_{\eta} \\
\omega_{\alpha} \downarrow & \omega_{\alpha+\delta} \downarrow \\
\mathcal{M}_{0}^{1, \alpha}(I)_{\eta} \times \mathbb{R}^{+} \stackrel{\mathcal{K}_{0}}{\longrightarrow} \mathcal{M}_{0}^{1, \alpha+\delta}(I)_{\eta} \times \mathbb{R}^{+} .
\end{array}
$$

By Lemma $2.2, \mathcal{K}_{0}\left(f_{0}, c\right)=\left(\mathcal{L}\left(f_{0}-1\right)+\gamma, \gamma / c^{1-\delta}\right)$ is a continuous map from $\mathcal{M}_{0}^{1, \alpha}(I)_{\eta} \times \mathbb{R}^{+}$to $\mathcal{M}_{0}^{1, \alpha+\delta}(I)_{\eta} \times \mathbb{R}^{+}$. Since the vertical arrows $\omega_{\alpha}, \omega_{\alpha+\delta}$ are homeomorphisms, $\mathcal{K}$ is a continuous map from $\mathcal{M}^{1, \alpha}(I)_{\eta}$ to $\mathcal{M}^{1, \alpha+\delta}(I)_{\eta}$.

To apply the implicit function theorem to $\mathcal{L} g=G$, we require the following:

Lemma 2.4. Let $0<\alpha<\alpha+\delta<1, \beta>0$ and let $0 \leq \eta \leq \alpha$. Then $\mathcal{L}^{\prime}(0)$ is $a$ homeomorphism of $\mathcal{C}^{1, \alpha}(I)_{\eta}$ onto $\mathcal{C}^{1, \alpha+\delta}(I)_{\eta}$.

Proof. We use the notation $\left(J_{\Phi} g\right)(x)=\int_{0}^{1} \Phi(t) g(x t) d t$. Then, by $(2.3),-\mathcal{L}^{\prime}(0)=$ $J_{\Phi_{0}}$, where

$$
\Phi_{0}(t)=(1-\delta) t^{\beta-1} \int_{0}^{t} \frac{d r}{\left(\int_{r}^{1} s^{\beta-1} d s\right)^{2-\delta}} .
$$

Moreover, by setting $\Phi(t):=t^{\eta} \Phi_{0}(t)$, we have the following commutative diagram:

$$
\begin{array}{ll}
\mathcal{C}^{1, \alpha}(I)_{0} \stackrel{J_{\Phi}}{\longrightarrow} \mathcal{C}^{1, \alpha+\delta}(I)_{0} \\
|x|^{\eta} \downarrow \\
\mathcal{C}^{1, \alpha}(I)_{\eta} \stackrel{-\mathcal{L}^{\prime}(0)}{\longrightarrow} \mathcal{C}^{1, \alpha+\delta}\left(\left.I\right|_{\eta} . \downarrow\right.
\end{array}
$$

where the vertical arrows $|x|^{\eta}$. are homeomorphisms. An elementary calculation shows that

$$
\Phi(t)=A t^{\beta}\left(1-t^{\beta}\right)^{\delta-1}+R(t), \quad A=\beta^{1-\delta}
$$

with $R(t)$ satisfying the estimates

$$
|R(t)| \leq M,\left|R^{\prime}(t)\right| \leq M t^{-1}(1-t)^{\delta-1},\left|R^{\prime \prime}(t)\right| \leq M t^{-2}(1-t)^{\delta-2},
$$


with some constant $M$. This enables us to employ Theorem 3.1 in Iwasaki-Kamimura [13], which tells us that $J_{\Phi}$ is a homeomorphism of $\mathcal{C}^{1, \alpha}(I)_{0}$ onto $\mathcal{C}^{1, \alpha+\delta}(I)_{0}$ if

$$
D_{\Phi}(z):=\int_{0}^{1} \Phi(t) t^{z} d t \neq 0,
$$

in the half plane $\operatorname{Re} z \geq 0$. Note that the space $\mathcal{C}^{k, \alpha}(I)_{0}$ in this paper is the same space as $\mathcal{C}^{k, \alpha}(I)$ in [13].

But, for $\Phi(t)=t^{\eta} \Phi_{0}(t)$, we have

$$
\begin{aligned}
D_{\Phi}(z) & =(1-\delta) \int_{0}^{1} t^{\eta+\beta+z-1} d t \int_{0}^{t} \frac{d r}{\left(\int_{r}^{1} s^{\beta-1} d s\right)^{2-\delta}} \\
& =(1-\delta) \int_{0}^{1} \frac{d r}{\left(\int_{r}^{1} s^{\beta-1} d s\right)^{2-\delta}} \int_{r}^{1} t^{\eta+\beta+z-1} d t \\
& =\frac{1-\delta}{\eta+\beta+z} \beta^{2-\delta} \int_{0}^{1} \frac{1-r^{\eta+\beta+z}}{\left(1-r^{\beta}\right)^{2-\delta}} d r,
\end{aligned}
$$

which does not vanish if $\operatorname{Re} z \geq 0$, because

$$
\operatorname{Re}\left(1-r^{\eta+\beta+z}\right)=1-r^{\eta+\beta+\operatorname{Re} z} \cos ((\operatorname{Im} z) \log r)>0
$$

for $r \in[0,1)$. Thus $J_{\Phi}$ is a homeomorphism of $\mathcal{C}^{1, \alpha}(I)_{0}$ onto $\mathcal{C}^{1, \alpha+\delta}(I)_{0}$, and so, by the commutative diagram, $-\mathcal{L}^{\prime}(0)$ is a homeomorphism of $\mathcal{C}^{1, \alpha}(I)_{\eta}$ onto $\mathcal{C}^{1, \alpha+\delta}(I)_{\eta}$.

Lemmas 2.2 and 2.4 allow us to use the implicit function theorem (see, e.g., Schwartz [28, Theorem 1.20]). We draw the following conclusion:

Lemma 2.5. Under the same assumption as in Lemma 2.4, the transform $\mathcal{L}$ maps a sufficiently small neighborhood $U$ of 0 in $\mathcal{C}^{1, \alpha}(I)_{\eta}$ homeomorphically onto a neighborhood $V$ of 0 in $\mathcal{C}^{1, \alpha+\delta}(I)_{\eta}$. This map $\mathcal{L}: U \rightarrow V$ is a $C^{1}$-diffeomorphism.

We are now in a position to give:

Proof of Proposition 2.1. Let $F$ be a function in $\mathcal{M}^{1, \alpha+\delta}(I)_{\eta}$ with $F(0)=\gamma$, where $\gamma$ is a number given in (2.1). Then the equation $\mathcal{K} f=F$ for $f \in \mathcal{M}^{1, \alpha}(I)_{\eta}$ is equivalent to $\mathcal{L}(f-1)=F-\gamma$ for $f-1 \in U$. We set $G:=F-\gamma$. Then $G \in \mathcal{C}^{1, \alpha+\delta}(I)_{\eta}, \gamma+G(x)>0$ on $I$.

We employ a scaling operator $S_{\kappa}$ for $\kappa>0$ defined by $\left(S_{\kappa} \phi\right)(x)=\phi(\kappa x)$. We view $S_{\kappa}$ as an operator from $\mathcal{C}^{1, v}\left(I_{\kappa}\right)_{\eta}$ onto $\mathcal{C}^{1, v}(I)_{\eta}, v=\alpha, \alpha+\delta$. Since 
$\mathcal{L} S_{\kappa}=S_{\kappa} \mathcal{L}$, we have the following commutative diagram:

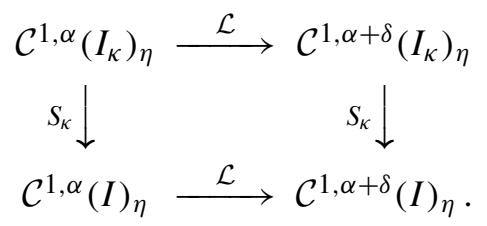

We denote the norm of $\phi \in \mathcal{C}^{1, v}\left(I_{\kappa}\right)_{\eta}$ by $\|\phi\|_{1, v, \eta ; \kappa}$. Then $S_{\kappa}$ has the norming property: $\left\|S_{\kappa} \phi\right\|_{1, v, \eta}=\kappa^{\eta}\|\phi\|_{1, v, \eta ; \kappa}$. Hence the vertical arrows in the above diagram are homeomorphisms. Notice that $S_{\kappa}^{-1}=S_{\kappa^{-1}}$ (see Figure 2.1).

Let $G^{\kappa}$ denote the section of $G:=F-\gamma$ on $I_{\kappa}$. Applying $S_{\kappa}$ to $G^{\kappa}$, we have

$$
\left\|S_{\kappa} G^{\kappa}\right\|_{1, \alpha+\delta, \eta}=\kappa^{\eta}\left\|G^{\kappa}\right\|_{1, \alpha+\delta, \eta ; \kappa} .
$$

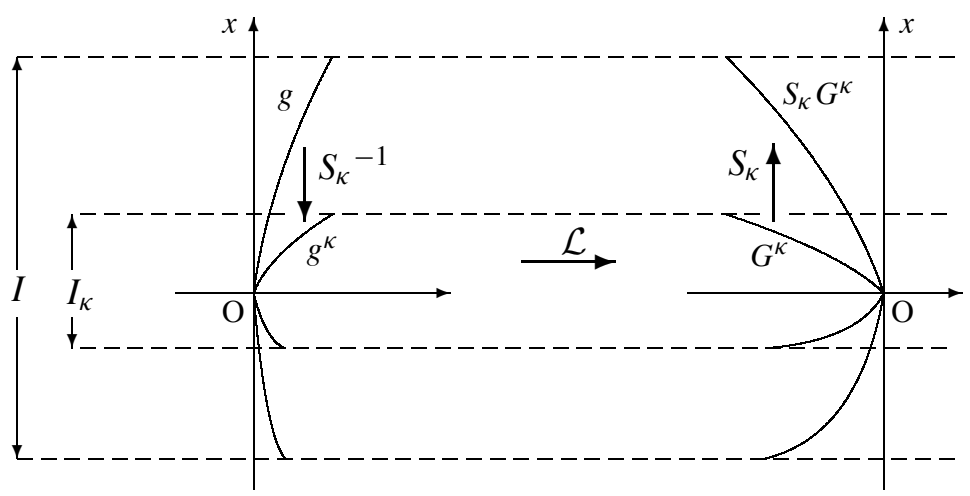

Figure 2.1. Diagram of the proof.

Since $\left\|G^{\kappa}\right\|_{1, \alpha+\delta, \eta ; \kappa}$ is not greater than the original norm $\|G\|_{1, \alpha+\delta, \eta}$ of $G$ in $\mathcal{C}^{1, \alpha+\delta}(I)_{\eta}$, if we take $\kappa>0$ sufficiently small, then the norm $\left\|S_{\kappa} G^{\kappa}\right\|_{1, \alpha+\delta, \eta}$ is so small that $S_{\kappa} G^{\kappa}$ is in the small neighborhood $V$ stated in Lemma 2.5. (Note that we assume $\eta>0$.) Hence, by the lemma, there exists a solution $g \in \mathcal{C}^{1, \alpha}(I)_{\eta}$ of $\mathcal{L} g=S_{\kappa} G^{\kappa}$, and hence the pull-back $g^{\kappa}:=S_{\kappa}{ }^{-1} g$, which belongs $\mathcal{C}^{1, \alpha}\left(I_{\kappa}\right)_{\eta}$, satisfies $\mathcal{L} g^{\kappa}=G$ on $I_{\kappa}$, or equivalently, $\mathcal{K}\left(1+g^{\kappa}\right)=F$ on $I_{\kappa}$. Thus we get a solution $f^{\kappa}:=1+g^{\kappa} \in \mathcal{M}^{1, \alpha}(I)_{\eta}$ of $\mathcal{K} f=F$ on $I_{\kappa}$.

We turn to the proof of assertion (2). Assume that there exist solutions $f_{i}-1 \in$ $\mathcal{C}^{1, \alpha}(I)_{\eta}, i=1,2$, of $\mathcal{L}(f-1)=F-\gamma$ on $I$ and let $f_{i}^{\kappa}$ and $F^{\kappa}$ be the sections of $f_{i}$ and $F$ on $I_{\kappa}$ respectively. Then $\mathcal{L}\left(f_{i}^{\kappa}-1\right)=F^{\kappa}-\gamma$ on $I_{\kappa}$, and hence $\mathcal{L}\left(S_{\kappa}\left(f_{i}^{\kappa}-1\right)\right)=S_{\kappa}\left(F^{\kappa}-\gamma\right), i=1,2$. If we take $\kappa>0$ sufficiently small then $S_{\kappa}\left(f_{i}^{\kappa}-1\right) \in U$ and $S_{\kappa}\left(F^{\kappa}-\gamma\right) \in V$, because $\left\|S_{\kappa}\left(f_{i}^{\kappa}-1\right)\right\|_{1, \alpha, \eta}=\kappa^{\eta} \| f_{i}^{\kappa}-$ $1\left\|_{1, \alpha, \eta ; \kappa} \leq \kappa^{\eta}\right\| f_{i}-1 \|_{1, \alpha, \eta}$ and $\left\|S_{\kappa}\left(F^{\kappa}-\gamma\right)\right\|_{1, \alpha+\delta, \eta}=\kappa^{\eta}\left\|F^{\kappa}-\gamma\right\|_{1, \alpha+\delta, \eta ; \kappa} \leq$ 
$\kappa^{\eta}\|F-\gamma\|_{1, \alpha+\delta, \eta}$. Therefore, by Lemma 2.5, we have $S_{\kappa}\left(f_{1}^{\kappa}-1\right)=S_{\kappa}\left(f_{2}^{\kappa}-1\right)$. This shows that $f_{1}^{\kappa}=f_{2}^{\kappa}$, that is, $f_{1}=f_{2}$ on $I_{\kappa}$. The proof is complete.

We conclude this section with the following:

Remark 2.6. Proposition 2.1 remains true even though the symbols $\mathcal{M}^{1, v}, v=$ $\alpha, \alpha+\delta$ are replaced by $\mathcal{M}^{0, v}, v=\alpha, \alpha+\delta$ respectively. In other words, the local solvability and uniqueness of the equation $\mathcal{K} f=F$ in the sense of the assertions in Proposition 2.1 still hold for the transform $\mathcal{K}: \mathcal{M}^{0, \alpha}(I) \rightarrow \mathcal{M}^{0, \alpha+\delta}(I)$. We omit the proof of this fact, because it is obtained by simply tracing the proof of Proposition 2.1.

\section{Fractional calculus}

We view $\mathcal{K}$ as a transform from $\mathcal{M}^{1, \alpha}(I)_{\eta}$ to $\mathcal{M}^{1, \alpha+\delta}(I)_{\eta}$, where $I$ is a bounded, closed interval containing 0 . However, since $\phi \in \mathcal{C}^{1, \alpha}(I)_{\eta}$ if and only if sections $\phi_{ \pm}$of $\phi$ on $I_{ \pm}:=I \cap \mathbb{R}_{ \pm}$belong to $\mathcal{C}^{1, \alpha}\left(I_{ \pm}\right)_{\eta}$ respectively, it suffices to treat the case either $I=I_{+}$or $I=I_{-}$; here and hereafter, we treat the case $I=[0, B]$, $B>0$. Throughout this section, for $b>0, C_{+}[0, b]$ denotes the set of positive, continuous functions on $[0, b]$.

A crucial characteristic of the equation $\mathcal{K} f=F$, namely,

$$
\int_{0}^{1} \frac{d t}{\left(\int_{t}^{1} s^{\beta-1} f(x s) d s\right)^{1-\delta}}=F(x),
$$

is that a solution $f$ remains in the space $\mathcal{M}^{1, \alpha}[0, b]_{\eta}$ as long as $F$ lives in $\mathcal{M}^{1, \alpha+\delta}[0, b]_{\eta}$, which enables us to obtain an extension of a local solution we obtained in the previous section. To describe the characteristic, it is convenient to adopt the following

Definition 3.1. A function $f$ is said to be regular on $[0, b]$ if it satisfies

$$
f \in C_{+}[0, b] \cap C^{1}(0, b], \quad|\theta f|_{\eta}<\infty \text { with some } \eta>0 .
$$

Moreover, $f$ is said to be a regular function on $[0, b)$ if it is regular on $\left[0, b^{\prime}\right]$ for any $b^{\prime}<b$. A function $f$ is said to be a regular solution on $[0, b]$ of $\mathcal{K} f=F$ if it is regular on $[0, b]$ and satisfies $(3.1)$ on $[0, b]$. A regular function on $[0, b)$ that satisfies $\mathcal{K} f=F$ is said to be a regular solution on $[0, b)$.

Our goal in this section is the following:

Proposition 3.2. Let $0<\alpha<\alpha+\delta<1, \beta>0$ and suppose that $F \in$ $\mathcal{M}^{1, \alpha+\delta}[0, b]_{0}$. Then:

(1) A regular solution $f$ on $[0, b]$ of $\mathcal{K} f=F$ belongs to $\mathcal{M}^{1, \alpha}[0, b]_{0}$. 
(2) If $f$ is a regular solution on $[0, b)$ of $\mathcal{K} f=F$, then there exists a regular solution $\tilde{f}$ on $[0, b]$ of $\mathcal{K} f=F$ such that $\tilde{f}=f$ on $[0, b)$.

Proposition 3.2 implies that a regular solution $f$ on $[0, b)$ of $\mathcal{K} f=F$ converges to a positive value and a function obtained by adding the value at $x=b$, which we denote by $\tilde{f}$, necessarily belongs to $\mathcal{M}^{1, \alpha}[0, b]_{0}$. The proof of this proposition is divided into several steps and will be completed only after Lemma 3.10.

The equation $\mathcal{K} f=F$ is rewritten as

$$
\int_{0}^{x} \frac{d \xi}{\left(\int_{\xi}^{x} r^{\beta-1} f(r) d r\right)^{1-\delta}}=x^{1-\beta+\beta \delta} F(x) .
$$

In view of this recast, for $a \geq 0$, we introduce a fractional integral operator $I_{a, w}^{\delta}$ of the following form:

$$
\left(I_{a, w}^{\delta} \phi\right)(x)=\frac{1}{\Gamma(\delta)} \int_{a}^{x} \frac{\phi(\xi)}{\left(\int_{\xi}^{x} r^{\beta-1} f(r) d r\right)^{1-\delta}} \xi^{\beta-1} f(\xi) d \xi,
$$

where $\Gamma(\cdot)$ is the Gamma function. Then (3.3) can be written as

$$
I_{0, w}^{\delta} \frac{1}{x^{\beta-1} f(x)}=\frac{1}{\Gamma(\delta)} x^{1-\beta+\beta \delta} F(x) .
$$

The operator $I_{a, w}^{\delta}$ is a fractional integral by a weight function $w(x)=\int_{a}^{x} r^{\beta-1} f(r) d r$ (refer to Samko-Kilbas-Marichev [26, Section 18.2]). The reason for $a \geq 0$ is the term $r^{\beta-1}$. Let $I_{a}^{\delta}$ be the Riemann-Liouville operator:

$$
\left(I_{a}^{\delta} \phi\right)(x)=\frac{1}{\Gamma(\delta)} \int_{a}^{x} \frac{\phi(\xi)}{(x-\xi)^{1-\delta}} d \xi .
$$

Then, provided that $f>0$, the operator $I_{a, w}^{\delta}$ may be connected with the RiemannLiouville operator $I_{a}^{\delta}$ by the relation $I_{a, w}^{\delta}=Q I_{0}^{\delta} Q^{-1}$ in terms of a substitution operator $Q$ defined by

$$
(Q \phi)(x)=\phi(w(x)), \quad w(x)=\int_{a}^{x} r^{\beta-1} f(r) d r .
$$

Hence, characteristics of the fractional integral operator $I_{a, w}^{\delta}$ are passed on from those of the Riemann-Liouville operator $I_{0}^{\delta}$. In particular, the inverse of $I_{a, w}^{\delta}$ is given by the associated differential operator $D_{a, w}^{\delta}$ defined as

$$
D_{a, w}^{\delta}=D_{w} I_{a, w}^{1-\delta}, \quad D_{w}=\frac{1}{x^{\beta-1} f(x)} \frac{d}{d x},
$$

in an appropriate framework. Some remarks on these fractional operators may be helpful at this stage: 
Lemma 3.3. Assume that $f \in C_{+}[a, b) \cap L^{1}(a, b)$ and let $I_{a, w}^{\delta}, D_{a, w}^{\delta}$ be operators defined by (3.4), (3.7). Then:

(1) If $\delta_{1}, \delta_{2}>0$ then $I_{a, w}^{\delta_{1}} I_{a, w}^{\delta_{2}} \phi=I_{a, w}^{\delta_{1}+\delta_{2}} \phi$ for $\phi \in C[a, b]$.

(2) If $0<\delta<1$ then

$$
\left(D_{a, w}^{\delta} \phi\right)(x)=\frac{1}{\Gamma(1-\delta)}\left\{\frac{\phi(a)}{\left(\int_{a}^{x} r^{\beta-1} f(r) d r\right)^{\delta}}+\int_{a}^{x} \frac{\phi^{\prime}(\xi)}{\left(\int_{\xi}^{x} r^{\beta-1} f(r) d r\right)^{\delta}} d \xi\right\}
$$

for $\phi \in A C[a, b]$. Here $A C[a, b]$ denotes the set of absolutely continuous functions on $[a, b]$.

(3) If $\phi \in L_{w}^{1}(a, b)$, namely, $\int_{a}^{b}|\phi(x)| w^{\prime}(x) d x<\infty$, and if $\phi \in C(a, b]$, then

$$
\lim _{\delta \rightarrow+0}\left(I_{a, w}^{\delta} \phi\right)(x)=\phi(x)
$$

at each $x \in(a, b]$.

(4) If $0<\delta<1$ then $I_{a, w}^{\delta} \phi \in C_{w}^{0, \delta}[a, b]$ for $\phi \in C[a, b]$. Here $C_{w}^{0, \delta}[a, b]$ is $a$ weighted Hölder space defined by

$$
C_{w}^{0, \delta}[a, b]:=\left\{\phi \in C[a, b]: \sup _{a \leq x \neq y \leq b} \frac{|\phi(x)-\phi(y)|}{|w(x)-w(y)|^{\delta}}<\infty\right\} .
$$

(5) If $0<\varepsilon<\delta+\varepsilon<1$ then $D_{a, w}^{1-\varepsilon} I_{a, w}^{\delta} \phi=D_{a, w}^{1-(\delta+\varepsilon)} \phi$ for $\phi \in C_{w}^{0,1-\delta}[a, b]$.

(6) If $0<\delta<\delta+\varepsilon<1$ then

$$
\begin{aligned}
\left(D_{a, w}^{\delta} \phi\right)(x) & =\frac{1}{\Gamma(1-\delta)} \frac{\phi(x)}{\left(\int_{a}^{x} r^{\beta-1} f(r) d r\right)^{\delta}} \\
& +\frac{\delta}{\Gamma(1-\delta)} \int_{a}^{x} \frac{\phi(x)-\phi(\xi)}{\left(\int_{\xi}^{x} r^{\beta-1} f(r) d r\right)^{1+\delta}} \xi^{\beta-1} f(\xi) d \xi, \quad a<x \leq b
\end{aligned}
$$

for $\phi \in C_{w}^{0, \delta+\varepsilon}[a, b]$.

Proof. All the assertions are rewritings of well-known facts via the operation $Q$ in (3.6):

(1) follows from the semigroup property $I_{a}^{\delta_{1}} I_{a}^{\delta_{2}} \phi=I_{a}^{\delta_{1}+\delta_{2}} \phi$ for $\phi \in C[a, b]$ (see, e.g., [26, equation (2.21)]) of $I_{a}^{\delta}$;

(2) is a recast of a formula ( [26, Lemma 2.2]) for a solution of the Abel's integral equation;

(3) follows from a well-known result [26, Theorem 2.7] for $I_{a}^{\delta}$. 
(4) is a recast of the fact ( [26, Corollary 2 of Theorem 3.1]) that $I_{a}^{\delta}$ is a bounded operator from $L^{\infty}(a, b)$ into the Hölder space $C^{0, \delta}[a, b]$;

(5) follows from (3.7) and (1) as $D_{a, w}^{1-\varepsilon} I_{a, w}^{\delta} \phi=D_{w} I_{a, w}^{\varepsilon} I_{a, w}^{\delta} \phi=D_{w} I_{a, w}^{\delta+\varepsilon} \phi=$ $D_{a, w}^{1-(\delta+\varepsilon)} \phi$

(6) is known as the Marchaud fractional derivative (see [26, Section 13 and equation (18.30)]).

Lemma 3.3 is standard. We need another lemma concerning mapping properties of the operator $I_{0, w}^{\delta}$.

Lemma 3.4. Let $0<\alpha<\alpha+\delta<1, \beta>0$. Then we have the followings:

(1) If $f \in C_{+}[0, b]$ then the operator $I_{0, w}^{\delta}$ is a homeomorphism of $\mathcal{C}^{0, \alpha}[0, b]_{1-\beta}$ onto $\mathcal{C}^{0, \alpha+\delta}[0, b]_{1-\beta+\beta \delta}$. The inverse is given by $D_{0, w}^{\delta}$, which is expressed in the Marchaud form

$$
\begin{aligned}
\left(D_{0, w}^{\delta} \phi\right)(x)= & \frac{1}{\Gamma(1-\delta)} \frac{\phi(x)}{\left(\int_{0}^{x} r^{\beta-1} f(r) d r\right)^{\delta}} \\
& +\frac{\delta}{\Gamma(1-\delta)} \int_{0}^{x} \frac{\phi(x)-\phi(\xi)}{\left(\int_{\xi}^{x} r^{\beta-1} f(r) d r\right)^{\delta+1}} \xi^{\beta-1} f(\xi) d \xi
\end{aligned}
$$

for $\phi \in \mathcal{C}^{0, \alpha+\delta}[0, b]_{1-\beta+\beta \delta .}$

(2) If $f$ is a regular function on $[0, b]$, then the operator $I_{0, w}^{\delta}$ is a homeomorphism of $\mathcal{C}^{1, \alpha}[0, b]_{1-\beta}$ onto $\mathcal{C}^{1, \alpha+\delta}[0, b]_{1-\beta+\beta \delta}$. The inverse is given by $D_{0, w}^{\delta}$.

Proof. We first prove the assertion (2). Let $\rho>-1$, put $b_{Q}:=\int_{0}^{b} r^{\beta-1} f(r) d r$, and set $\Phi(t)=t^{\rho}(1-t)^{\delta-1} / \Gamma(\delta)$. Then it follows from [13, Theorem 3.1] that an operator $J_{\Phi}$ defined by $\left(J_{\Phi} g\right)(x)=\int_{0}^{1} \Phi(t) g(x t) d t$ is a homeomorphism of $\mathcal{C}^{1, \alpha}\left[0, b_{Q}\right]_{0}$ onto $\mathcal{C}^{1, \alpha+\delta}\left[0, b_{Q}\right]_{0}$, since $\int_{0}^{1} \Phi(t) t^{z} d t=B(\rho+z+1, \delta) / \Gamma(\delta)$, where $B(\cdot, \cdot)$ denotes the beta function, has no zeros in $\operatorname{Re} z \geq 0$ in view of $\rho>-1$. Accordingly, by the commutative diagram

$$
\begin{array}{ccc}
\mathcal{C}^{1, \alpha}\left[0, b_{Q}\right]_{0} & \stackrel{J_{\Phi}}{\longrightarrow} & \mathcal{C}^{1, \alpha+\delta}\left[1, b_{Q}\right]_{0} \\
|x|^{\rho} . & |x|^{\rho+\delta} . \\
\mathcal{C}^{1, \alpha}\left[0, b_{Q}\right]_{\rho} & \stackrel{I_{0}^{\delta}}{\longrightarrow} & \mathcal{C}^{1, \alpha+\delta}\left[0, b_{Q}\right]_{\rho+\delta},
\end{array}
$$


$I_{0}^{\delta}$ is a homeomorphism of $\mathcal{C}^{1, \alpha}\left[0, b_{Q}\right]_{\rho}$ onto $\mathcal{C}^{1, \alpha+\delta}\left[0, b_{Q}\right]_{\rho+\delta}$. The inverse $\left(I_{0}^{\delta}\right)^{-1}$ of $I_{0}^{\delta}$ is given by

$$
\left(I_{0}^{\delta}\right)^{-1} \phi(x)=\frac{1}{\Gamma(1-\delta)} \frac{\phi(x)}{x^{\delta}}+\frac{\delta}{\Gamma(1-\delta)} \int_{0}^{x} \frac{\phi(x)-\phi(\xi)}{(x-\xi)^{1+\delta}} d \xi
$$

for $\phi \in \mathcal{C}^{1, \alpha+\delta}\left[0, b_{Q}\right]_{\rho+\delta}$ (see [26, equation (13.2)]).

We now let $Q$ be an operator defined by (3.6). Then it follows from an elementary estimation that, for each $\rho \in \mathbb{R}$, the operator $Q$ is a homeomorphism of $\mathcal{C}^{1, \alpha}\left[0, b_{Q}\right]_{\rho}$ onto $\mathcal{C}^{1, \alpha}[0, b]_{\beta \rho}$ provided that $f$ is a regular function on $[0, b]$. Hence we have the commutative diagram

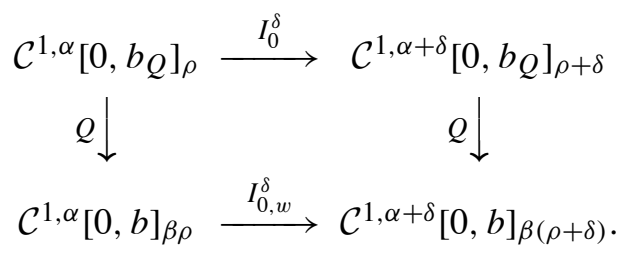

Here the top and vertical arrows are homeomorphisms provided that $\rho>-1$, and so is the bottom arrow. Accordingly, by setting $\rho=\beta^{-1}-1$, we conclude that $I_{0, w}^{\delta}$ is a homeomorphism of $\mathcal{C}^{1, \alpha}[0, b]_{1-\beta}$ onto $\mathcal{C}^{1, \alpha+\delta}[0, b]_{1-\beta+\beta \delta}$. The expression (3.8) follows from (3.9) via $Q$ in (3.6). We have completed the proof of the assertion (2). Following the same steps as above, with $\mathcal{C}^{0, v}$ instead of $\mathcal{C}^{1, v}, v=\alpha, \alpha+\delta$, we may prove the assertion (1). Unlike (2), no condition other than $f \in C_{+}[0, b]$ need be imposed on $f$.

We now return to the equation $\mathcal{K} f=F$. By means of Lemma 3.4 we can derive its differential form of the order $\delta$, which is useful in studying a priori properties of its solutions:

Lemma 3.5. Let $0<\alpha<\alpha+\delta<1, \beta>0$. Then we have the followings:

(1) Under the assumption $F \in \mathcal{C}^{0, \alpha+\delta}[0, b]_{0}$, a regular function $f$ on $[0, b]$ satisfies $\mathcal{K} f=F$ on $[0, b]$ if and only if $f$ satisfies

$$
\begin{aligned}
\frac{\pi}{\sin \pi \delta} \frac{1}{f(x)}= & \frac{F(x)}{\left(\int_{0}^{1} s^{\beta-1} f(x s) d s\right)^{\delta}} \\
& +\delta \int_{0}^{1} \frac{F(x)-t^{1-\beta+\beta \delta} F(x t)}{\left(\int_{t}^{1} s^{\beta-1} f(x s) d s\right)^{\delta+1}} t^{\beta-1} f(x t) d t
\end{aligned}
$$

on $[0, b]$. 
(2) Under the assumption $F \in \mathcal{C}^{1, \alpha+\delta}[0, b]_{0}$, if $f$ is a regular solution on $[0, b]$ of $\mathcal{K} f=F$ orland (3.11), then $f(x)$ belongs to $\mathcal{C}^{1, \alpha}[0, b]_{0}$. In this case, for each $a \in(0, b]$, the equation $\mathcal{K} f=F$ on $[a, b]$ is equivalent to

$$
\begin{aligned}
\frac{\pi}{\sin \pi \delta} \frac{1}{f(x)}= & \frac{x^{\beta \delta} F(x)}{\left(\int_{0}^{x} r^{\beta-1} f(r) d r\right)^{\delta}}-x^{\beta-1} \frac{x^{1-\beta+\beta \delta} F(x)-a^{1-\beta+\beta \delta} F(a)}{\left(\int_{a}^{x} r^{\beta-1} f(r) d r\right)^{\delta}} \\
& +\delta \int_{0}^{a} \frac{\xi^{\beta-1} x^{\beta \delta} F(x)-x^{\beta-1} \xi^{\beta \delta} F(\xi)}{\left(\int_{\xi}^{x} r^{\beta-1} f(r) d r\right)^{\delta+1}} f(\xi) d \xi \\
& +x^{\beta-1} \int_{a}^{x} \frac{\frac{d}{d \xi}\left(\xi^{1-\beta+\beta \delta} F(\xi)\right)}{\left(\int_{\xi}^{x} r^{\beta-1} f(r) d r\right)^{\delta}} d \xi .
\end{aligned}
$$

Proof. As is already noted, (3.1) is written as (3.5). Suppose that a regular function $f$ on $[0, b]$ satisfies $(3.1)$ on $[0, b]$. Then, by the estimate

$$
\begin{aligned}
\left|\frac{x^{\alpha}}{f(x)}-\frac{y^{\alpha}}{f(y)}\right| & =\left|\int_{y}^{x} \frac{\xi^{\alpha-1}(\alpha f(\xi)-(\theta f)(\xi))}{f(\xi)^{2}} d \xi\right| \\
& \leq \exists M_{1}\left|\int_{y}^{x} \xi^{\alpha-1} d \xi\right| \leq M_{2}|x-y|^{\alpha},
\end{aligned}
$$

$f(x)^{-1}$ belongs to $\mathcal{C}^{0, \alpha}[0, b]_{0}$ for each $\alpha \in(0,1)$, and hence (3.5) holds in the framework stated in (1) of Lemma 3.4. Hence, applying the operator $D_{0, w}^{\delta}$ in Lemma 3.4 to (3.5), we find that (3.1) is equivalent to

$$
f(x)^{-1}=\frac{1}{\Gamma(\delta)} x^{\beta-1} D_{0, w}^{\delta} x^{1-\beta+\beta \delta} F(x) .
$$

By means of (3.8) and $\Gamma(\delta) \Gamma(1-\delta)=\pi / \sin \pi \delta$, this equality can be rewritten as

$$
\begin{aligned}
\frac{\pi}{\sin \pi \delta} \frac{1}{f(x)}= & \frac{x^{\beta \delta} F(x)}{\left(\int_{0}^{x} r^{\beta-1} f(r) d r\right)^{\delta}} \\
& +\delta \int_{0}^{x} \frac{\xi^{\beta-1} x^{\beta \delta} F(x)-x^{\beta-1} \xi^{\beta \delta} F(\xi)}{\left(\int_{\xi}^{x} r^{\beta-1} f(r) d r\right)^{\delta+1}} f(\xi) d \xi
\end{aligned}
$$

The corresponding substitutions show that this is equivalent to (3.11). If $F \in$ $\mathcal{C}^{1, \alpha+\delta}[0, b]_{0}$ then, by Lemma 3.4.(2), $f(x)^{-1}$ belongs to $\mathcal{C}^{1, \alpha}[0, b]_{0}$. This, together with $f \in C_{+}[0, b]$, yields $|\theta f|_{0}<\infty,|\theta f|_{\alpha, 0}<\infty$. Hence $f \in \mathcal{C}^{1, \alpha}[0, b]_{0}$.

Conversely, if a regular function $f$ on $[0, b]$ satisfies $(3.11)$, then we have

$$
\frac{1}{x^{\beta-1} f(x)}=\frac{1}{\Gamma(\delta)} D_{0, w^{\delta}}^{x^{1-\beta+\beta \delta}} F(x), \quad 0 \leq x \leq b .
$$


Applying $I_{0, w}^{\delta}$ to this equality, we arrive at (3.5).

We may rewrite (3.14) as

$$
\begin{aligned}
\frac{\pi}{\sin \pi \delta} \frac{1}{f(x)}= & \frac{x^{\beta \delta} F(x)}{\left(\int_{0}^{x} r^{\beta-1} f(r) d r\right)^{\delta}} \\
& +\delta \int_{0}^{a} \frac{\xi^{\beta-1} x^{\beta \delta} F(x)-x^{\beta-1} \xi^{\beta \delta} F(\xi)}{\left(\int_{\xi}^{x} r^{\beta-1} f(r) d r\right)^{\delta+1}} f(\xi) d \xi \\
& +\delta \int_{a}^{x} \frac{\xi^{\beta-1} x^{\beta \delta} F(x)-x^{\beta-1} \xi^{\beta \delta} F(\xi)}{\left(\int_{\xi}^{x} r^{\beta-1} f(r) d r\right)^{\delta+1}} f(\xi) d \xi, \quad a \leq x \leq b .
\end{aligned}
$$

If $F \in \mathcal{C}^{1, \alpha+\delta}[0, b]_{0}$ then, by an integration by parts, we have

$$
\begin{aligned}
& \delta \int_{a}^{x} \frac{\xi^{\beta-1} x^{\beta \delta} F(x)-x^{\beta-1} \xi^{\beta \delta} F(\xi)}{\left(\int_{\xi}^{x} r^{\beta-1} f(r) d r\right)^{\delta+1}} f(\xi) d \xi \\
& \quad=-x^{\beta-1} \frac{x^{1-\beta+\beta \delta} F(x)-a^{1-\beta+\beta \delta} F(a)}{\left(\int_{a}^{x} r^{\beta-1} f(r) d r\right)^{\delta}}+x^{\beta-1} \int_{a}^{x} \frac{\left(\xi^{1-\beta+\beta \delta} F(\xi)\right)^{\prime}}{\left(\int_{\xi}^{x} r^{\beta-1} f(r) d r\right)^{\delta}} d \xi
\end{aligned}
$$

for $0<a \leq x \leq b$. Hence we obtain (3.12).

We give three remarks regarding Lemma 3.5: (1) Lemma 3.5.(2) tells us that if $F \in \mathcal{M}^{1, \alpha+\delta}[0, b]_{0}$ then a regular solution $f$ on $[0, b]$ of $\mathcal{K} f=F$ necessarily belongs to $\mathcal{M}^{1, \alpha}[0, b]_{0}$. When we know a priori the solution $f$ to be in $\mathcal{M}^{1, \alpha}[0, a]_{\eta}$ with some $a>0$, this implies that $f \in \mathcal{M}^{1, \alpha}[0, b]_{\eta}$, since if a function $f \in \mathcal{M}^{1, \alpha}[0, b]_{0}$ belongs to $\mathcal{M}^{1, \alpha}[0, a]_{\eta}$ with some $a>0$ then $f$ belongs to $\mathcal{M}^{1, \alpha}[0, b]_{\eta}$; (2) in the case where $1-\beta+\beta \delta \geq 0$, equation (3.12) becomes much simpler because we can take $a=0$; (3) equation (3.12) is a representation of (3.13) via the Marchaud form. Hence, by Lemma 3.4.(2), the function in the right-hand belongs to $\mathcal{C}^{1, \alpha}[0, b]_{0}$ provided that $f$ is a regular function on $[0, b]$ and $F \in \mathcal{C}^{1, \alpha+\delta}[0, b]_{0}$.

In what follows we shall study behaviors at $b$ of regular solutions on $[0, b)$ of $\mathcal{K} f=F$. We begin with the following lemma, which states that each regular solution on $[0, b)$ of $\mathcal{K} f=F$ keeps away from zero.

Lemma 3.6. Let $0<\alpha<\alpha+\delta<1, \beta>0$, suppose that $F \in \mathcal{M}^{1, \alpha+\delta}[0, b]_{0}$, and let $f$ be a regular solution on $[0, b)$ of $\mathcal{K} f=F$. Then $\inf _{0 \leq x<b} f(x)>0$.

Proof. We fix numbers $a, d$ so that $0<a<d<b$ and let $b^{\prime}$ be a number in $(d, b)$. By using Lemma 3.5.(2) with any $b^{\prime}$ in $(a, b)$ in place of $b$ in the lemma, it follows 
that $f$ satisfies (3.12) for $a \leq x<b^{\prime}$. Hence we have

$$
\begin{aligned}
\frac{\pi}{\sin \pi \delta} \frac{1}{f(x)} \leq & \frac{x^{\beta \delta}|F(x)|}{\left(\int_{0}^{d} r^{\beta-1} f(r) d r\right)^{\delta}}+x^{\beta-1} \frac{x^{1-\beta+\beta \delta} F(x)+a^{1-\beta+\beta \delta} F(a)}{\left(\int_{a}^{d} r^{\beta-1} f(r) d r\right)^{\delta}} \\
& +\delta \int_{0}^{a} \frac{\left|\xi^{\beta-1} x^{\beta \delta} F(x)-x^{\beta-1} \xi^{\beta \delta} F(\xi)\right|}{\left(\int_{a}^{d} r^{\beta-1} f(r) d r\right)^{\delta+1}} f(\xi) d \xi \\
& +x^{\beta-1} \int_{a}^{x} \frac{\left|\frac{d}{d \xi}\left(\xi^{1-\beta+\beta \delta} F(\xi)\right)\right|}{\left(\int_{\xi}^{x} r^{\beta-1} f(r) d r\right)^{\delta}} d \xi
\end{aligned}
$$

for $d \leq x \leq b^{\prime}$. It follows from this inequality that

$$
\frac{\pi}{\sin \pi \delta} \frac{1}{f(x)} \leq A_{0}+x^{\beta-1} \int_{a}^{x} \frac{\left|\frac{d}{d \xi}\left(\xi^{1-\beta+\beta \delta} F(\xi)\right)\right|}{\left(\int_{\xi}^{x} r^{\beta-1} f(r) d r\right)^{\delta}} d \xi, \quad d \leq x \leq b^{\prime}
$$

with some constant $A_{0}$. This constant is independent of $b^{\prime}$, since $F \in \mathcal{M}^{1, \alpha+\delta}[0, b]_{0}$. In addition, for $d \leq x \leq b^{\prime}$,

$$
\begin{aligned}
\int_{a}^{x} \frac{\left|\frac{d}{d \xi}\left(\xi^{1-\beta+\beta \delta} F(\xi)\right)\right|}{\left(\int_{\xi}^{x} r^{\beta-1} f(r) d r\right)^{\delta}} d \xi= & \int_{a}^{d} \frac{\left|\frac{d}{d \xi}\left(\xi^{1-\beta+\beta \delta} F(\xi)\right)\right|}{\left(\int_{\xi}^{x} r^{\beta-1} f(r) d r\right)^{\delta}} d \xi \\
& +\int_{d}^{x} \frac{\left|\frac{d}{d \xi}\left(\xi^{1-\beta+\beta \delta} F(\xi)\right)\right|}{\left(\int_{\xi}^{x} r^{\beta-1} f(r) d r\right)^{\delta}} d \xi \\
\leq & \int_{a}^{d} \frac{\left|\frac{d}{d \xi}\left(\xi^{1-\beta+\beta \delta} F(\xi)\right)\right|}{\left(\int_{\xi}^{d} r^{\beta-1} f(r) d r\right)^{\delta}} d \xi \\
& +\int_{d}^{x} \frac{\left|\frac{d}{d \xi}\left(\xi^{1-\beta+\beta \delta} F(\xi)\right)\right|}{\left(\int_{\xi}^{x} r^{\beta-1} d r\right)^{\delta}} d \xi\left(\min _{d \leq x \leq b^{\prime}} f(x)\right)^{-\delta} .
\end{aligned}
$$

Hence there exist positive constants $A_{1}, A_{2}$ independent of $b^{\prime}$ such that

$$
\frac{1}{f(x)} \leq A_{1}+A_{2}\left(\min _{d \leq x \leq b^{\prime}} f(x)\right)^{-\delta}, \quad d \leq x \leq b^{\prime} .
$$

By $\min _{d \leq x \leq b^{\prime}} f(x) \leq f(x)$ for $d \leq x \leq b^{\prime}$, we arrive at

$$
1 \leq A_{1}\left(\min _{d \leq x \leq b^{\prime}} f(x)\right)+A_{2}\left(\min _{d \leq x \leq b^{\prime}} f(x)\right)^{1-\delta} .
$$


Since $A_{1} X+A_{2} X^{1-\delta}$ approaches 0 as $X \rightarrow 0$, this implies that there exists a positive constant $m$ independent of $b^{\prime}$ such that $m \leq \min _{d \leq x \leq b^{\prime}} f(x)$. This proves the lemma.

Hereafter we use the notation: $m_{f}=\inf _{0 \leq x<b} f(x)$. By Lemma 3.6, $m_{f}$ is a positive number. With the aid of this fact we can draw the following conclusion:

Lemma 3.7. Under the same assumptions as in Lemma 3.6, there exists a limit of $f(x)^{-1}$ as $x \rightarrow b:$

$$
\exists \lim _{x \rightarrow b} \frac{1}{f(x)}<\infty
$$

Proof. We take a number $a$ in $(0, b)$ and let $x \rightarrow b$ in (3.12). Since $\left(\int_{*}^{x} r^{\beta-1} f(r) d r\right)^{-\delta}$ is monotonically decreasing, the first three terms in the right-hand side in (3.12) converge as $x \rightarrow b$. Hence the proof of the lemma is reduced to showing that the fourth term there converges as $x \rightarrow b$. We rewrite it as

$$
x^{\beta-1} \int_{a}^{x} \frac{\frac{d}{d \xi}\left(\xi^{1-\beta+\beta \delta} F(\xi)\right)}{\left(\int_{\xi}^{x} r^{\beta-1} f(r) d r\right)^{\delta}} d \xi=x^{\beta \delta} \int_{a / x}^{1} \frac{\frac{d}{d t}\left(t^{1-\beta+\beta \delta} F(x t)\right)}{\left(\int_{x t}^{x} r^{\beta-1} f(r) d r\right)^{\delta}} d t
$$

for $a \leq x<b$. It follows from the assumption $F \in \mathcal{M}^{1, \alpha+\delta}[0, b]_{0}$ that

$$
\left|\frac{\frac{d}{d t}\left(t^{1-\beta+\beta \delta} F(x t)\right)}{\left(\int_{x t}^{x} r^{\beta-1} f(r) d r\right)^{\delta}}\right| \leq \frac{M}{m_{f} \delta} \frac{1}{\left(\int_{x t}^{x} r^{\beta-1} d r\right)^{\delta}} \leq \frac{M_{1}}{\left(1-t^{\beta}\right)^{\delta}}
$$

for $a \leq x<b$. Here $M, M_{1}$ are constants independent of $x \in[a, b)$. This enables us to use Lebesgue's convergence theorem. Thus we have

$$
\lim _{x \rightarrow b} x^{\beta-1} \int_{a}^{x} \frac{\frac{d}{d \xi}\left(\xi^{1-\beta+\beta \delta} F(\xi)\right)}{\left(\int_{\xi}^{x} r^{\beta-1} f(r) d r\right)^{\delta}} d \xi=b^{\beta \delta} \int_{a / b}^{1} \frac{\frac{d}{d t}\left(t^{1-\beta+\beta \delta} F(b t)\right)}{\left(\int_{b t}^{b} r^{\beta-1} f(r) d r\right)^{\delta}} d t<\infty .
$$

The proof is complete.

We now turn to a question of whether it can happen that a solution $f$ of (3.1) blows up. Actually, $f$ does not blow up, namely, $f$ converges to a finite value as $x \rightarrow b$. To prove it we require the following preliminary lemma:

Lemma 3.8. Under the same assumptions as in Lemma 3.6,

$$
\int_{0}^{b} r^{\beta-1} f(r) d r<\infty
$$


Proof. We rewrite (3.3) as

$$
\int_{0}^{1} \frac{d t}{\left(\int_{x t}^{x} r^{\beta-1} f(r) d r\right)^{1-\delta}}=x^{-\beta+\beta \delta} F(x) .
$$

By Lemma 3.6, the integrand is estimated as, for $0<a \leq x \leq b$,

$$
\frac{1}{\left(\int_{x t}^{x} r^{\beta-1} f(r) d r\right)^{1-\delta}} \leq \frac{1}{m_{f}{ }^{1-\delta}\left(\int_{x t}^{x} r^{\beta-1} d r\right)^{1-\delta}} \leq \frac{\exists M}{\left(1-t^{\beta}\right)^{1-\delta}},
$$

where $M$ is a constant independent of $x$. Hence, by applying Lebesgue's convergence theorem to (3.15), we have

$$
\int_{0}^{1} \frac{d t}{\left(\int_{b t}^{b} r^{\beta-1} f(r) d r\right)^{1-\delta}}=b^{-\beta+\beta \delta} F(b) .
$$

Accordingly, if $\int_{0}^{b} r^{\beta-1} f(r) d r=\infty$ then $F(b)=0$. This contradicts the assumption $F(x)>0$ on $[0, b]$. Thus we have proved the lemma.

The following lemma assures that a solution $f(x)$ of (3.1) never blows up as $x$ tends to $b$ as long as $F \in \mathcal{M}^{1, \alpha+\delta}[0, b]_{0}$.

Lemma 3.9. Under the same assumptions as in Lemma 3.6, the solution $f$ converges to a finite value as $x \rightarrow b$ :

$$
\exists \lim _{x \rightarrow b} f(x)<\infty
$$

Proof. By Lemma 3.7, we have the alternative: either $f(x)$ converges to a finite value or $f(x)$ tends to $\infty$ as $x \rightarrow b$. We shall show that the latter case does not occur by contradiction; let us suppose that

$$
\lim _{x \rightarrow b} f(x)=\infty .
$$

We take $d$ so that $0<d<b$ and rewrite (3.3) as

$$
\int_{0}^{d} \frac{d \xi}{\left(\int_{\xi}^{x} r^{\beta-1} f(r) d r\right)^{1-\delta}}+\int_{d}^{x} \frac{d \xi}{\left(\int_{\xi}^{x} r^{\beta-1} f(r) d r\right)^{1-\delta}}=x^{1-\beta+\beta \delta} F(x)
$$

for $d \leq x<b$. Thus, by setting

$$
q(x):=\int_{0}^{d} \frac{d \xi}{\left(\int_{\xi}^{x} r^{\beta-1} f(r) d r\right)^{1-\delta}}, \quad d \leq x \leq b
$$


we have

$$
\int_{d}^{x} \frac{d \xi}{\left(\int_{\xi}^{x} r^{\beta-1} f(r) d r\right)^{1-\delta}}=x^{1-\beta+\beta \delta} F(x)-q(x), \quad d \leq x \leq b .
$$

This is written as

$$
I_{d, w}^{\delta} \frac{1}{x^{\beta-1} f(x)}=\frac{1}{\Gamma(\delta)}\left(x^{1-\beta+\beta \delta} F(x)-q(x)\right)
$$

in terms of the fractional integral operator $I_{d, w}^{\delta}$ defined in (3.4). Since

$$
q^{\prime}(x)=(\delta-1) \int_{0}^{d} \frac{d \xi}{\left(\int_{\xi}^{x} r^{\beta-1} f(r) d r\right)^{2-\delta}} x^{\beta-1} f(x)<0,
$$

we have $\int_{d}^{b}\left|q^{\prime}(x)\right| d x=q(d)-q(b)<\infty$. This implies that $q(x) \in A C[d, b]$. Therefore $x^{1-\beta+\beta \delta} F(x)-q(x) \in A C[d, b] \cap C^{1}(d, b]$.

We now let $0<\varepsilon<1-\delta$ and apply the fractional differential operator $D_{d, w}^{1-\varepsilon}$ defined in (3.7) to (3.17). Then

$$
D_{d, w}^{1-\varepsilon} I_{d, w}^{\delta} \frac{1}{x^{\beta-1} f(x)}=\frac{1}{\Gamma(\delta)} D_{d, w}^{1-\varepsilon}\left(x^{1-\beta+\beta \delta} F(x)-q(x)\right) .
$$

We shall first consider the limit as $\varepsilon \rightarrow 0$ of the right-hand side in (3.19) at $x=b$. By observing that $d^{1-\beta+\beta \delta} F(d)-q(d)=0$ and using Lemma 3.3.(2) it follows that

$$
\begin{aligned}
D_{d, w}^{1-\varepsilon}\left(x^{1-\beta+\beta \delta} F(x)-q(x)\right) & =\frac{1}{\Gamma(\varepsilon)} \int_{d}^{x} \frac{\left(\xi^{1-\beta+\beta \delta} F(\xi)-q(\xi)\right)^{\prime}}{\left(\int_{\xi}^{x} r^{\beta-1} f(r) d r\right)^{1-\varepsilon}} d \xi \\
& =I_{d, w}^{\varepsilon}\left\{\frac{\left(x^{1-\beta+\beta \delta} F(x)-q(x)\right)^{\prime}}{x^{\beta-1} f(x)}\right\}
\end{aligned}
$$

for $d \leq x \leq b$. We let $\varepsilon \rightarrow 0$. Then, by Lemma 3.3.(3), (3.16), (3.18), we obtain

$$
\begin{aligned}
\left.\lim _{\varepsilon \rightarrow 0} I_{d, w}^{\varepsilon}\left\{\frac{\left(x^{1-\beta+\beta \delta} F(x)-q(x)\right)^{\prime}}{x^{\beta-1} f(x)}\right\}\right|_{x=b} & =\left.\frac{\left(x^{1-\beta+\beta \delta} F(x)-q(x)\right)^{\prime}}{x^{\beta-1} f(x)}\right|_{x=b} \\
& =(1-\delta) \int_{0}^{d} \frac{d \xi}{\left(\int_{\xi}^{b} r^{\beta-1} f(r) d r\right)^{2-\delta}}>0 .
\end{aligned}
$$

This shows that

$$
\lim _{\varepsilon \rightarrow 0} \frac{1}{\Gamma(\delta)}\left(D_{d, w}^{1-\varepsilon}\left(x^{1-\beta+\beta \delta} F(x)-q(x)\right)\right)(b)>0 .
$$


We shall next consider the limit as $\varepsilon \rightarrow 0$ of the left-hand side in (3.19) at $x=b$. We employ (3.12) with $a \in(0, d)$ to show that $x^{1-\beta} f(x)^{-1}$ belongs to the weighted Hölder space $C_{w}^{0,1-\delta}[d, b]$ defined in Lemma 3.3.(4). Notice that (3.12) still holds at $x=b$ in view of Lemma 3.8. Moreover, by Lemma 3.8, we see that $\mid w(x)-$ $w(y) \mid \leq \int_{d}^{b} r^{\beta-1} f(r) d r=: M$ for any $x, y \in[d, b]$. Hence, by the estimation

$$
\begin{aligned}
& \left|\frac{1}{\left(\int_{0}^{x} r^{\beta-1} f(r) d r\right)^{\delta}}-\frac{1}{\left(\int_{0}^{y} r^{\beta-1} f(r) d r\right)^{\delta}}\right|=\left|\delta \int_{y}^{x} \frac{\xi^{\beta-1} f(\xi)}{\left(\int_{0}^{\xi} r^{\beta-1} f(r) d r\right)^{\delta+1}} d \xi\right| \\
& \leq \frac{\delta}{\left(\int_{0}^{d} r^{\beta-1} f(r) d r\right)^{\delta+1}}\left|\int_{y}^{x} \xi^{\beta-1} f(\xi) d \xi\right| \\
& \leq \frac{\delta}{\left(\int_{0}^{d} r^{\beta-1} f(r) d r\right)^{\delta+1}}|w(x)-w(y)|^{1-\delta} M^{\delta}, \quad d \leq x, y \leq b,
\end{aligned}
$$

we can show that the first term of the right-hand side in (3.12) belongs to the weighted Hölder space $C_{w}^{0,1-\delta}[d, b]$. In a similar way, we can verify that the second and third terms there also belong to $C_{w}^{0,1-\delta}[d, b]$. Moreover, by Lemma 3.3.(4), the fourth term belongs to $C_{w}^{0,1-\delta}[a, b]$ because it is written as

$$
x^{\beta-1} \Gamma(1-\delta) I_{a, w}^{1-\delta}\left(\frac{\left(x^{1-\beta+\beta \delta} F(x)\right)^{\prime}}{x^{\beta-1} f(x)}\right), \quad \text { where } \frac{\left(x^{1-\beta+\beta \delta} F(x)\right)^{\prime}}{x^{\beta-1} f(x)} \in C[a, b] .
$$

Thus the function $x^{1-\beta} f(x)^{-1}$ belongs to the weighted Hölder space $C_{w}^{0,1-\delta}[d, b]$, and hence, by using of Lemma 3.3.(5),(6), we obtain

$$
\begin{aligned}
& D_{d, w}^{1-\varepsilon} I_{d, w}^{\delta} \frac{1}{x^{\beta-1} f(x)}=D_{d, w}^{1-(\delta+\varepsilon)} \frac{1}{x^{\beta-1} f(x)} \\
& =\frac{1}{\Gamma(\delta+\varepsilon)} \frac{1}{\left(\int_{d}^{x} r^{\beta-1} f(r) d r\right)^{1-\delta-\varepsilon} x^{\beta-1} f(x)} \\
& \quad+\frac{1-\delta-\varepsilon}{\Gamma(\delta+\varepsilon)} \int_{d}^{x} \frac{\xi^{\beta-1} f(\xi)}{\left(\int_{\xi}^{x} r^{\beta-1} f(r) d r\right)^{2-\delta-\varepsilon}}\left(\frac{1}{x^{\beta-1} f(x)}-\frac{1}{\xi^{\beta-1} f(\xi)}\right) d \xi
\end{aligned}
$$

for $d<x \leq b$. This, combined with (3.16), yields

$$
\left(D_{d, w}^{1-\varepsilon} I_{d, w}^{\delta} \frac{1}{x^{\beta-1} f(x)}\right)(b)=\frac{\delta+\varepsilon-1}{\Gamma(\delta+\varepsilon)} \int_{d}^{b} \frac{d \xi}{\left(\int_{\xi}^{b} r^{\beta-1} f(r) d r\right)^{2-\delta-\varepsilon}} .
$$

This must converge to a positive value as $\varepsilon \rightarrow 0$ in view of (3.19), (3.20). But it is impossible because the right-hand side of the above equality is negative for $\delta+\varepsilon<1$. The proof is complete. 
By Lemmas 3.7 and 3.9, a regular solution $f$ on $[0, b)$ of $\mathcal{K} f=F$ converges to a finite, positive value as $x \rightarrow b$. Our next task is to prove the following:

Lemma 3.10. Under the same assumptions as in Lemma 3.6, the derivative $f^{\prime}(x)$ of the solution $f(x)$ converges to a finite value as $x \rightarrow b$.

Proof. Differentiating (3.11) we get

$$
\begin{aligned}
& -\frac{\pi}{\sin \pi \delta} \frac{f^{\prime}(x)}{f(x)^{2}}=\left(\frac{F(x)}{\left(\int_{0}^{1} s^{\beta-1} f(x s) d s\right)^{\delta}}\right)^{\prime} \\
& +\delta \int_{0}^{1} \frac{F(x)-t^{1-\beta+\beta \delta} F(x t)}{\left(\int_{t}^{1} s^{\beta-1} f(x s) d s\right)^{\delta+1}} t^{\beta} f^{\prime}(x t) d t \\
& -\delta(\delta+1) \int_{0}^{1} \frac{\int_{t}^{1} s^{\beta} f^{\prime}(x s) d s}{\left(\int_{t}^{1} s^{\beta-1} f(x s) d s\right)^{\delta+2}}\left(F(x)-t^{1-\beta+\beta \delta} F(x t)\right) t^{\beta-1} f(x t) d t \\
& +\delta \int_{0}^{1} \frac{F^{\prime}(x)-t^{2-\beta+\beta \delta} F^{\prime}(x t)}{\left(\int_{t}^{1} s^{\beta-1} f(x s) d s\right)^{\delta+1}} t^{\beta-1} f(x t) d t
\end{aligned}
$$

for $0<x<b$. The second term of the right-hand side is rewritten as

$$
\delta x^{\beta-2} \int_{0}^{x} \frac{\int_{\xi}^{x}\left(r^{1-\beta+\beta \delta} F(r)\right)^{\prime} d r}{\left(\int_{\xi}^{x} r^{\beta-1} f(r) d r\right)^{\delta+1}} \xi^{\beta} f^{\prime}(\xi) d \xi .
$$

Also, by an interchange of the order of integration, the third term is rewritten as

$$
-\delta(\delta+1) x^{\beta-2} \int_{0}^{x} \xi^{\beta} f^{\prime}(\xi) d \xi \int_{0}^{\xi} \frac{\int_{\tau}^{x}\left(r^{1-\beta+\beta \delta} F(r)\right)^{\prime} d r}{\left(\int_{\tau}^{x} r^{\beta-1} f(r) d r\right)^{\delta+2}} \tau^{\beta-1} f(\tau) d \tau .
$$

Therefore, by setting

$$
\begin{aligned}
& K(x, \xi)=-\frac{\sin \pi \delta}{\pi} x^{\beta-2} f(x)^{2} \xi^{\beta} \times \\
& \left\{\delta \frac{\int_{\xi}^{x}\left(r^{1-\beta+\beta \delta} F(r)\right)^{\prime} d r}{\left(\int_{\xi}^{x} r^{\beta-1} f(r) d r\right)^{\delta+1}}-\delta(\delta+1) \int_{0}^{\xi} \frac{\int_{\tau}^{x}\left(r^{1-\beta+\beta \delta} F(r)\right)^{\prime} d r}{\left(\int_{\tau}^{x} r^{\beta-1} f(r) d r\right)^{\delta+2}} \tau^{\beta-1} f(\tau) d \tau\right\}, \\
& p(x):=-\frac{\sin \pi \delta}{\pi} f(x)^{2} \times \\
& \left\{\left(\frac{x^{\beta \delta} F(x)}{\left(\int_{0}^{x} r^{\beta-1} f(r) d r\right)^{\delta}}\right)^{\prime}+\delta \int_{0}^{1} \frac{F^{\prime}(x)-t^{2-\beta+\beta \delta} F^{\prime}(x t)}{\left(\int_{t}^{1} s^{\beta-1} f(x s) d s\right)^{\delta+1}} t^{\beta-1} f(x t) d t\right\},
\end{aligned}
$$


we have

$$
f^{\prime}(x)-\int_{0}^{x} K(x, \xi) f^{\prime}(\xi) d \xi=p(x), \quad 0<x<b .
$$

Let $a$ be fixed in $(0, b)$. By Lemmas 3.7 and 3.9, we may view $f$ as a function in $C_{+}[0, b]$. Hence we get the estimate $|K(x, \xi)| \leq M(x-\xi)^{-\delta}$ for $a \leq x \leq b$, $\xi<x$ with a constant $M$ independent of $x, \xi$. Moreover, by the assumption $F \in$ $\mathcal{M}^{1, \alpha+\delta}[0, b]_{0}$ with $\alpha>0$, we can show that $p \in C[a, b]$. Therefore $p_{1}(x):=$ $p(x)+\int_{0}^{a} K(x, \xi) f^{\prime}(\xi) d \xi$ belongs to $C[a, b]$. In this way we arrive at

$$
f^{\prime}(x)=p_{1}(x)+\int_{a}^{x} K(x, \xi) f^{\prime}(\xi) d \xi, \quad a \leq x<b,
$$

where $K(x, \xi)$ satisfies $|K(x, \xi)| \leq M(x-\xi)^{-\delta}$. By setting $L:=\max _{a \leq x \leq b} p_{1}(x)$ this yields

$$
\left|f^{\prime}(x)\right| \leq L+M \int_{a}^{x} \frac{\left|f^{\prime}(\xi)\right|}{(x-\xi)^{\delta}} d \xi, \quad a \leq x<b .
$$

From this it is readily seen by the Gronwall inequality that $\sup _{a \leq x<b}\left|f^{\prime}(x)\right|<\infty$. Hence, by letting $x \rightarrow b$ in (3.21), it follows that $f^{\prime}(x)$ converges to a finite value as $x \rightarrow b$. The proof is complete.

We are now in a position to give:

Proof of Proposition 3.2. A function $f$ belongs to $\mathcal{M}^{1, \alpha}[0, b]_{0}$ if (and only if) $f$ is positive and belongs to $\mathcal{C}^{1, \alpha}[0, b]_{0}$. Hence assertion (1) is immediate from Lemma 3.5.(2). By the assumption in the proposition and Definition 3.1, $f$ satisfies (3.1) for $0 \leq x<b$, and so, by Lemma 3.5, satisfies (3.12) for $0 \leq x<b$. Hence, by Lemmas 3.7 and 3.9, the solution $f$ converges to a finite, positive value $f_{b}$. This implies that a natural extension $\tilde{f}$ that is defined by just setting $\tilde{f}(b)=f_{b}$ in addition to $\tilde{f}(x)=f(x)$ for $0 \leq x<b$ belongs to $C_{+}[0, b]$. But, by Lemma $3.10, f^{\prime}(x)$ also converges to a finite value. This implies that $\tilde{f} \in C^{1}(0, b]$, as is immediately checked by the mean value theorem. Thus $\tilde{f}$ is a regular solution on $[0, b]$ of $\mathcal{K} f=F$.

\section{Extension}

In the previous section we have shown that each regular solution on $[0, b)$ of $\mathcal{K} f=$ $F$ can be extended to a regular solution on $[0, b]$ of the equation. In this section we shall show that the solution can be extended beyond $b$. Our goal in this section is to show that $\mathcal{K}: \mathcal{M}^{1, \alpha}(I)_{\eta} \rightarrow \mathcal{M}^{1, \alpha+\delta}(I)_{\eta}$ is a bijection (Proposition 4.3).

We adopt the following:

Definition 4.1. A solution $f_{1}$ of $\mathcal{K} f=F$ is said to be a regular extension on $\left[0, b_{1}\right]$ of solution $f$ if $f$ is a regular solution on $[0, b], f_{1}$ is a regular solution on $\left[0, b_{1}\right]$, $b<b_{1}$, and $f_{1}(x)=f(x)$ on $[0, b]$. 
Then we have:

Proposition 4.2. Let $F \in \mathcal{M}^{1, \alpha+\delta}[0, B]_{0}$, let $0<b<B$, and let $f(x)$ be $a$ regular solution of $\mathcal{K} f=F$ on $[0, b]$. Then there exist a number $b_{1} \in(b, B]$ and a regular extension $f_{1}$ on $\left[0, b_{1}\right]$ of $f$. This extension is unique; if $f_{2}$ is a regular extension on $\left[0, b_{2}\right]$, then $f_{2}(x)=f_{1}(x)$ for $0 \leq x \leq \min \left(b_{1}, b_{2}\right)$.

Proof. We proceed in four steps.

Step 1. By Lemma 3.5, the equation $\mathcal{K} f=F$ on $[0, B]$ is equivalent to (3.11) on $[0, B]$. We shall prove the proposition by applying the implicit function theorem to (3.11). Let $f_{L}(x)$ be a linear function in the interval $[b, B]$ defined by

$$
f_{L}(x):=f(b)+f^{\prime}(b)(x-b) .
$$

We take a number $c \in(b, B]$ so that $f_{L}(x)>0$ in $[b, c]$ and define a function $f_{c}$ on $[0, c]$ by

$$
f_{c}(x):= \begin{cases}f(x) & \text { for } 0 \leq x \leq b \\ f_{L}(x) & \text { for } b \leq x \leq c .\end{cases}
$$

Then $f_{c}$ is a regular function on $[0, c]$. Next we define a function $\Lambda$ on $[b, c]$ by

$$
\Lambda(x):=f_{L}(x)\left\{\frac{F(x)}{\left(\int_{0}^{1} s^{\beta-1} f_{c}(x s) d s\right)^{\delta}}+\delta \int_{0}^{1} \frac{F(x)-t^{1-\beta+\beta \delta} F(x t)}{\left(\int_{t}^{1} s^{\beta-1} f_{c}(x s) d s\right)^{\delta+1}} t^{\beta-1} f_{c}(x t) d t\right\} .
$$

By remark (3) just after the proof of Lemma 3.5, the function $\Lambda(x)$ belongs to the Hölder space $C^{1, \alpha}[b, c]$. In particular $\Lambda(x) \in C^{1}[b, c]$. Moreover we have $\Lambda(b)=\frac{\pi}{\sin \pi \delta}, \Lambda^{\prime}(b)=0$, since $f_{L}(b)=f(b), f_{L}^{\prime}(b)=f^{\prime}(b)$ and $f(x)$ satisfies (3.11) on $[0, b]$. In view of $\Lambda(b)>0$, we may assume that $\Lambda(x)>0$ on $[b, c]$ by retaking $c$ near $b$ if need be. In what follows let both $f_{L}(x)$ and $\Lambda(x)$ be positive on $[b, c]$. (See Figure 4.1.)

Let $C_{0}^{1}[b, c]$ be a Banach space defined by

$$
C_{0}^{1}[b, c]:=\left\{h \in C^{1}[b, c]: h(b)=h^{\prime}(b)=0\right\}
$$

with the norm

$$
\|h\|:=\max _{b \leq x \leq c}\left|h^{\prime}(x)\right|,
$$

and consider a mapping $\mathcal{T}$ on $C_{0}^{1}[b, c]$ defined by

$$
\begin{aligned}
& (\mathcal{T}(h))(x):=\left(f_{L}(x)+h(x)\right)\left\{\frac{F(x)}{\left(\int_{0}^{1} s^{\beta-1}\left(f_{c}(x s)+\tilde{h}(x s)\right) d s\right)^{\delta}}\right. \\
& \left.\quad+\delta \int_{0}^{1} \frac{F(x)-t^{1-\beta+\beta \delta} F(x t)}{\left(\int_{t}^{1} s^{\beta-1}\left(f_{c}(x s)+\tilde{h}(x s)\right) d s\right)^{\delta+1}} t^{\beta-1}\left(f_{c}(x t)+\tilde{h}(x t)\right) d t\right\}-\Lambda(x),
\end{aligned}
$$


where

$$
\tilde{h}(x):= \begin{cases}0 & \text { for } 0 \leq x \leq b, \\ h(x) & \text { for } b \leq x \leq c .\end{cases}
$$

If $h \in C_{0}^{1}[b, c]$ then $\tilde{h} \in C^{1}[0, c]$ and $\tilde{h}(x)=\tilde{h}^{\prime}(x)=0$ on $[0, b]$. Hence $(\mathcal{T}(h))(b)=(\mathcal{T}(h))^{\prime}(b)=0$ for each $h \in C_{0}^{1}[b, c]$. Thus $\mathcal{T}$ maps $C_{0}^{1}[b, c]$ into itself. Moreover $\mathcal{T}(0)=0$. Notice that (3.11) is rewritten as $\mathcal{T}(h)=\frac{\pi}{\sin \pi \delta}-\Lambda(x)$.

As is easily verified, $\mathcal{T}$ is a $C^{1}$-mapping of an open neighborhood of 0 in $C_{0}^{1}[b, c]$ into $C_{0}^{1}[b, c]$. The Fréchet derivative $\mathcal{T}^{\prime}(0)$ at 0 of $\mathcal{T}$ is given as

$$
\left(\mathcal{T}^{\prime}(0) h\right)(x)=\frac{\Lambda(x)}{f_{L}(x)}\left\{h(x)-\int_{b}^{x} L(x, \xi) h(\xi) d \xi\right\}
$$

for $h \in C_{0}^{1}[b, c]$, where

$$
\begin{array}{r}
L(x, \xi):=\delta \frac{f_{L}(x)^{2}}{\Lambda(x)}\left\{\frac{\xi^{\beta-1} x^{\beta \delta} F(x)}{\left(\int_{0}^{x} r^{\beta-1} f_{c}(r) d r\right)^{\delta+1}}-\frac{\xi^{\beta-1} x^{\beta \delta} F(x)-x^{\beta-1} \xi^{\beta \delta} F(\xi)}{\left(\int_{\xi}^{x} r^{\beta-1} f_{c}(r) d r\right)^{\delta+1}}\right. \\
\left.+(\delta+1) \xi^{\beta-1} \int_{0}^{\xi} \frac{s^{\beta-1} x^{\beta \delta} F(x)-x^{\beta-1} s^{\beta \delta} F(s)}{\left(\int_{s}^{x} r^{\beta-1} f_{c}(r) d r\right)^{\delta+2}} f_{c}(s) d s\right\}, \quad b \leq \xi<x \leq c .
\end{array}
$$

By the assumption $F \in \mathcal{M}^{1, \alpha+\delta}[0, B]_{0}, F$ belongs to $C^{1}[b, c]$. Hence there exists a constant $M_{0}$ independent of $x, \xi$ such that $|L(x, \xi)| \leq M_{0}(x-\xi)^{-\delta}$.

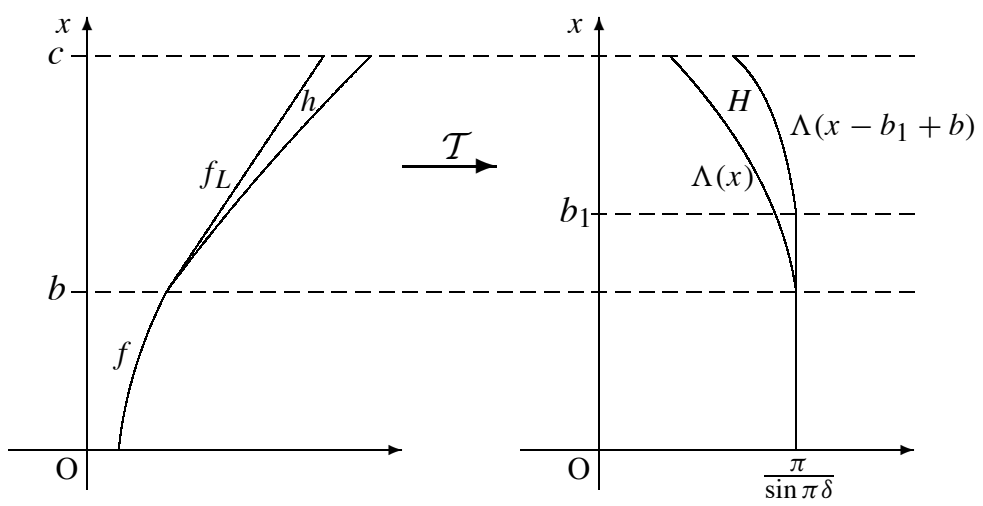

Figure 4.1. Diagram of the proof.

Step 2. Given $H(x) \in C_{0}^{1}[b, c]$, we consider $\mathcal{T}^{\prime}(0) h=H$, which is written as

$$
h(x)-\int_{b}^{x} L(x, \xi) h(\xi) d \xi=\frac{f_{L}(x)}{\Lambda(x)} H(x), \quad b \leq x \leq c .
$$


We set $L_{1}(x, \xi):=\int_{\xi}^{x} L(x, z) d z$. Then, for $h \in C_{0}^{1}[b, c]$, equation (4.2) is rewritten as

$$
h(x)-\int_{b}^{x} L_{1}(x, \xi) h^{\prime}(\xi) d \xi=\frac{f_{L}(x)}{\Lambda(x)} H(x), \quad b \leq x \leq c .
$$

Observing that

$$
\begin{gathered}
\int_{\xi}^{x} \frac{z^{\beta-1} x^{\beta \delta} F(x)-x^{\beta-1} z^{\beta \delta} F(z)}{\left(\int_{z}^{x} r^{\beta-1} f_{c}(r) d r\right)^{\delta+1}} d z=\int_{\xi / x}^{1} \frac{t^{\beta-1} F(x)-t^{\beta \delta} F(x t)}{\left(\int_{t}^{1} s^{\beta-1} f_{c}(x s) d s\right)^{\delta+1}} d t \\
\int_{\xi}^{x} z^{\beta-1} d z \int_{0}^{z} \frac{s^{\beta-1} x^{\beta \delta} F(x)-x^{\beta-1} s^{\beta \delta} F(s)}{\left(\int_{s}^{x} r^{\beta-1} f_{c}(r) d r\right)^{\delta+2}} f_{c}(s) d s \\
=\int_{\xi}^{x} z^{\beta-1} d z\left(\int_{0}^{\xi}+\int_{\xi}^{z}\right) \cdots d s \\
=\frac{1}{\beta}\left(x^{\beta}-\xi^{\beta}\right) \int_{0}^{\xi} \frac{s^{\beta-1} x^{\beta \delta} F(x)-x^{\beta-1} s^{\beta \delta} F(s)}{\left(\int_{s}^{x} r^{\beta-1} f_{c}(r) d r\right)^{\delta+2}} f_{c}(s) d s \\
+\frac{1}{\beta} \int_{\xi / x}^{1} \frac{\left(1-t^{\beta}\right)\left(t^{\beta-1} F(x)-t^{\beta \delta} F(x t)\right)}{\left(\int_{t}^{1} s^{\beta-1} f_{c}(x s) d s\right)^{\delta+2}} f_{c}(x t) d t
\end{gathered}
$$

and noting that $F \in \mathcal{M}^{1, \alpha+\delta}[0, b]_{0}$ with $\alpha>0$, we obtain the estimate $\left|\frac{\partial}{\partial x} L_{1}(x, \xi)\right| \leq$ $M_{1}(x-\xi)^{-\delta}$ with a constant $M_{1}$. Hence, differentiating (4.3) and setting $L_{2}(x, \xi):=$ $\frac{\partial}{\partial x} L_{1}(x, \xi)$, we get

$$
h^{\prime}(x)-\int_{b}^{x} L_{2}(x, \xi) h^{\prime}(\xi) d \xi=\frac{d}{d x}\left(\frac{f_{L}(x)}{\Lambda(x)} H(x)\right), \quad b \leq x \leq c .
$$

The kernel $L_{2}(x, \xi)$ satisfies $\left|L_{2}(x, \xi)\right| \leq M_{2}(x-\xi)^{-\delta}$ with some constant $M_{2}$. Moreover the right-hand side of this equation belongs to the Banach space

$$
C_{0}^{0}[b, c]:=\{h \in C[b, c]: h(b)=0\}
$$

equipped with the norm $\|h\|:=\max _{b \leq x \leq c}|h(x)|$. Hence, by a standard method of successive approximation for the Volterra equation of the second kind, equation (4.5) has a unique solution $h^{\prime}$ in $C_{0}^{0}[b, c]$. Moreover, as is easily verified by integrating (4.5) from $b$ to $x$, the function $h(x):=\int_{b}^{x} h^{\prime}(y) d y \in C_{0}^{1}[b, c]$ satisfies (4.3), and hence (4.2). This shows that $\mathcal{T}^{\prime}(0): C_{0}^{1}[b, c] \rightarrow C_{0}^{1}[b, c]$ has a bounded linear inverse. Hence, by the implicit function theorem, $\mathcal{T}$ maps a sufficiently small neighborhood $U$ of 0 in $C_{0}^{1}[b, c]$ homeomorphically onto a neighborhood $V$ of 0 in $C_{0}^{1}[b, c]$. 
Step 3. For $p \in(b, c]$ we define a function $H_{p}$ on $[b, c]$ by

$$
H_{p}(x):= \begin{cases}\frac{\pi}{\sin \pi \delta}-\Lambda(x) & \text { for } b \leq x \leq p \\ \Lambda(x-p+b)-\Lambda(x) & \text { for } p \leq x \leq c\end{cases}
$$

Then $H_{p} \in C_{0}^{1}[b, c]$ by virtue of $\Lambda(b)=\frac{\pi}{\sin \pi \delta}, \Lambda^{\prime}(b)=0$. Recalling that $\Lambda \in$ $C^{1, \alpha}[b, c]$ we have

$$
\begin{aligned}
\left\|H_{p}\right\|=\sup _{b \leq x \leq c}\left|H_{p}^{\prime}(x)\right| & =\sup _{b \leq x \leq p}\left|\Lambda^{\prime}(x)\right|+\sup _{p \leq x \leq b}\left|\Lambda^{\prime}(x-p+b)-\Lambda^{\prime}(x)\right| \\
& \leq \sup _{b \leq x \leq p}\left|\Lambda^{\prime}(x)\right|+\left(\sup _{b \leq x \neq y \leq c} \frac{\left|\Lambda^{\prime}(x)-\Lambda^{\prime}(y)\right|}{|x-y|^{\alpha}}\right)(p-b)^{\alpha},
\end{aligned}
$$

which tends to 0 as $p$ approaches $b$ because of $\Lambda^{\prime}(b)=0$. Accordingly there exists a number $b_{1} \in(b, c]$ such that $H_{b_{1}}(x)$ is in the neighborhood $V$ stated above. For this function $H=H_{b_{1}}(x)$, the equation $\mathcal{T}(h)=H$ has a solution $h$ in the neighborhood $U$. Since $H_{b_{1}}(x)=\frac{\pi}{\sin \pi \delta}-\Lambda(x)$ for $b \leq x \leq b_{1}$, the solution $h$ satisfies $\mathcal{T}(h)=\frac{\pi}{\sin \pi \delta}-\Lambda(x)$ for $b \leq x \leq b_{1}$.

We finally set

$$
f_{1}(x):= \begin{cases}f(x) & \text { for } 0 \leq x \leq b \\ f_{L}(x)+h(x) & \text { for } b \leq x \leq b_{1}\end{cases}
$$

Then $f_{1}$ is a regular function on $\left[0, b_{1}\right]$. By $\mathcal{T}(h)=\frac{\pi}{\sin \pi \delta}-\Lambda(x)$ for $b \leq x \leq b_{1}$, the function $f_{1}$ satisfies $(3.11)$ on $\left[b, b_{1}\right]$. Thus $f_{1}$ is a regular extension on $\left[0, b_{1}\right]$ of $f$. We have proved the existence of a regular extension.

Step 4. We shall prove the uniqueness of the extension. Let $f_{i}, i=1,2$, be regular extensions on $\left[0, b_{i}\right]$ of $f$. Then $f_{2}(x)=f_{1}(x)$ for $0 \leq x \leq b$ and, by (3.14),

$$
\begin{aligned}
& f_{2}(x)\left\{\frac{x^{\beta \delta} F(x)}{\left(\int_{0}^{x} r^{\beta-1} f_{2}(r) d r\right)^{\delta}}+\delta \int_{0}^{x} \frac{\xi^{\beta-1} x^{\beta \delta} F(x)-x^{\beta-1} \xi^{\beta \delta} F(\xi)}{\left(\int_{\xi}^{x} r^{\beta-1} f_{2}(r) d r\right)^{\delta+1}} f_{2}(\xi) d \xi\right\} \\
& =f_{1}(x)\left\{\frac{x^{\beta \delta} F(x)}{\left(\int_{0}^{x} r^{\beta-1} f_{1}(r) d r\right)^{\delta}}+\delta \int_{0}^{x} \frac{\xi^{\beta-1} x^{\beta \delta} F(x)-x^{\beta-1} \xi^{\beta \delta} F(\xi)}{\left(\int_{\xi}^{x} r^{\beta-1} f_{1}(r) d r\right)^{\delta+1}} f_{1}(\xi) d \xi\right\}
\end{aligned}
$$

for $b \leq x \leq c$, where $c=\min \left(b_{1}, b_{2}\right)$. We set $f(\tau, x):=(1-\tau) f_{1}(x)+\tau f_{2}(x)$. 
Then we get

$$
\begin{aligned}
& \left(f_{2}(x)-f_{1}(x)\right)\left\{\frac{x^{\beta \delta} F(x)}{\left(\int_{0}^{x} r^{\beta-1} f_{2}(r) d r\right)^{\delta}}\right. \\
& \left.+\delta \int_{0}^{x} \frac{\xi^{\beta-1} x^{\beta \delta} F(x)-x^{\beta-1} \xi^{\beta \delta} F(\xi)}{\left(\int_{\xi}^{x} r^{\beta-1} f_{2}(r) d r\right)^{\delta+1}} f_{2}(\xi) d \xi\right\} \\
& =-f_{1}(x)\left\{\int_{0}^{1} \frac{d}{d \tau} \frac{x^{\beta \delta} F(x)}{\left(\int_{0}^{x} r^{\beta-1} f(\tau, r) d r\right)^{\delta}} d \tau\right. \\
& \left.+\delta \int_{0}^{1} \frac{d}{d \tau} \int_{0}^{x} \frac{\xi^{\beta-1} x^{\beta \delta} F(x)-x^{\beta-1} \xi^{\beta \delta} F(\xi)}{\left(\int_{\xi}^{x} r^{\beta-1} f(\tau, r) d r\right)^{\delta+1}} f(\tau, \xi) d \xi d \tau\right\} .
\end{aligned}
$$

Computing the right-hand side of this equality by an interchange of the order of integration, observing that the left-hand side of this equality equals to $\left(f_{2}(x)-\right.$ $\left.f_{1}(x)\right) \frac{\pi}{\sin \pi \delta} f_{2}(x)^{-1}$, and noting $f_{2}(x)-f_{1}(x)=0$ for $0 \leq x \leq b$, we obtain

$$
f_{2}(x)-f_{1}(x)=\int_{b}^{x} \Gamma_{0}(x, \xi)\left(f_{2}(\xi)-f_{1}(\xi)\right) d \xi, \quad b \leq x \leq c,
$$

where we define a function $\Gamma_{0}(x, \xi)$ on $b \leq \xi<x \leq c$ by

$$
\begin{aligned}
\Gamma_{0}(x, \xi): & =\delta \frac{\sin \pi \delta}{\pi} f_{1}(x) f_{2}(x) \times \\
& \left\{\int_{0}^{1} \frac{\xi^{\beta-1} x^{\beta \delta} F(x)}{\left(\int_{0}^{x} r^{\beta-1} f(\tau, r) d r\right)^{\delta+1}} d \tau-\int_{0}^{1} \frac{\xi^{\beta-1} x^{\beta \delta} F(x)-x^{\beta-1} \xi^{\beta \delta} F(\xi)}{\left(\int_{\xi}^{x} r^{\beta-1} f(\tau, r) d r\right)^{\delta+1}} d \tau\right. \\
& \left.+(\delta+1) \xi^{\beta-1} \int_{0}^{1} d \tau \int_{0}^{\xi} \frac{s^{\beta-1} x^{\beta \delta} F(x)-x^{\beta-1} s^{\beta \delta} F(s)}{\left(\int_{s}^{x} r^{\beta-1} f(\tau, r) d r\right)^{\delta+2}} f(\tau, s) d s\right\} .
\end{aligned}
$$

By noting that $f(\tau, x)$ is a positive function on $[0,1] \times[b, c]$ and that $F \in C^{1}[b, c]$, it follows that there exists a constant $M$ independent of $x, \xi$ such that $\left|\Gamma_{0}(x, \xi)\right| \leq$ $M(x-\xi)^{-\delta}$. Hence, by applying the Gronwall inequality to (4.6), we get $f_{2}(x)-$ $f_{1}(x)=0$ for each $x \in[b, c]$.

We are now in a position to establish the following:

Proposition 4.3. Under the same assumptions as in Theorem 1.1, the transform $\mathcal{K}: \mathcal{M}^{1, \alpha}(I)_{\eta} \rightarrow \mathcal{M}^{1, \alpha+\delta}(I)_{\eta}$ is a bijection. 
Proof. As was stated in the beginning of Section 3, it suffices to prove the proposition in the case $I=[0, B], B>0$. We shall first show that $\mathcal{K}$ is injective. To do so, let $f_{i} \in \mathcal{M}^{1, \alpha}[0, B]_{\eta}, i=1,2$, be solutions of $\mathcal{K} f=F$, where $F \in \mathcal{M}^{1, \alpha+\delta}[0, B]_{\eta}$. Then, by Proposition 2.1.(2), we get $f_{1}=f_{2}$ on $[0, \kappa B]$ for sufficiently small $\kappa>0$. Let $f_{0}$ denote this solution $f_{1}\left(=f_{2}\right)$ on $[0, \kappa B]$. Then $f_{1}, f_{2}$ are regular extensions on $[0, B]$ of $f_{0}$. Thus, by Proposition 4.2 , we conclude that $f_{1}=f_{2}$ on $[0, B]$.

We shall next show that $\mathcal{K}$ is surjective. To do so, given $F \in \mathcal{M}^{1, \alpha+\delta}[0, B]_{\eta}$, we define an interval $J \subset[0, B]$ by

$J:=\{b \in(0, B]:$ there exists a regular solution on $[0, b]$ of $\mathcal{K} f=F\}$

By Proposition 2.1.(1), we get a solution $f_{0} \in \mathcal{M}^{1, \alpha}[0, \kappa B]_{\eta}$ on $[0, \kappa B]$ of $\mathcal{K} f=$ $F$ provided that $\kappa>0$ is sufficiently small. Since a function $f \in \mathcal{M}^{1, \alpha}[0, b]_{\eta}$ with $\eta>0$ is a regular function on $[0, b]$, this solution is a regular solution on $[0, \kappa B]$ of the equation. This implies that $J$ is not empty; we put $b^{*}:=\sup J$. Then, for any $b^{\prime}<b^{*}$, there exists $b^{\prime \prime} \in\left(b^{\prime}, b^{*}\right)$ for which we have a regular solution on $\left[0, b^{\prime \prime}\right]$ of the equation. We define $f(x)$ on $\left[0, b^{\prime}\right]$ as the section of the solution on $\left[0, b^{\prime}\right]$. By the uniqueness of the extensions, this definition is independent of the particular point $b^{\prime \prime} \in\left(b^{\prime}, b^{*}\right)$. Thus we get a regular solution $f$ on $\left[0, b^{*}\right)$ of $\mathcal{K} f=F$. But, by Proposition 3.2.(2), this solution is extended to a regular solution on $\left[0, b^{*}\right]$ of the equation. If $b^{*}<B$ then, by Proposition 4.2, this regular solution on $\left[0, b^{*}\right]$ is further extended beyond $b^{*}$, contradicting the definition of $b^{*}$. This implies that $b^{*}=B$. By repeating the above argument at $b^{*}$ it follows that there exists a regular solution $f$ on $[0, B]$ of $\mathcal{K} f=F$. But, by Proposition 3.2.(1), this solution $f$ must belong to $\mathcal{M}^{1, \alpha}[0, B]_{0}$. By the uniqueness of the extensions, this solution is an extension of $f_{0}$ on $[0, \kappa B]$, which belongs to $\mathcal{M}^{1, \alpha}[0, \kappa B]_{\eta}$. This shows that $f \in \mathcal{M}^{1, \alpha}[0, B]_{\eta}$. The proof is complete.

Remark 4.4. An inspection to Step 4 in the proof of Proposition 4.2 shows that the assumptions $f_{1}, f_{2} \in C[b, c]$ and $F \in C^{1}[b, c]$ with $c=\min \left(b_{1}, b_{2}\right)$ are enough for the uniqueness of the extensions. This, combined with the fact described in Remark 2.6, yields the following conclusion: if $f_{1}, f_{2} \in \mathcal{M}^{0, \alpha}(I)_{\eta}$ satisfy $\mathcal{K} f=F$ for $F \in \mathcal{M}^{1, \alpha+\delta}(I)_{\eta}$, then $f_{1}(x)=f_{2}(x)$ for any $x \in I$.

We conclude this section with an example:

Example 4.5. Let $\beta=1$ and consider the function $f_{\mu}(x)=(1-x)^{\mu}$ on $[0,1]$. Note that this function belongs to $\mathcal{M}^{1, \alpha}[0, B]_{\eta}$ for any positive $B<1$ and any $\alpha, \eta$ such that $0<\eta \leq \alpha<1-\delta$, while it does not belong to $\mathcal{M}^{1, \alpha}[0,1]_{\eta}$ unless $\mu=0$; if $\mu>0$ then $f_{\mu}(x)$ is not positive at $x=1$ and, by contraries, if $\mu<0$ then $f_{\mu}(x)$ diverges to $+\infty$ as $x \rightarrow 1$ : unless $\mu=0$, the function $f_{\mu}$ has the point $x=1$ as a singularity in the sense that either $f_{\mu}(1)=\infty$ or $f_{\mu}(1)=0$. 
We shall first treat the case where $\mu>-1$. Then we get

$$
\begin{aligned}
\left(\mathcal{K} f_{\mu}\right)(x) & =(1+\mu)^{1-\delta} \int_{0}^{1}\left(\frac{x}{(1-x t)^{1+\mu}-(1-x)^{1+\mu}}\right)^{1-\delta} d t \\
& =(1+\mu)^{1-\delta} \int_{0}^{1}\left(\frac{1-\left(\frac{1-x}{1-x t}\right)}{1-\left(\frac{1-x}{1-x t}\right)^{1+\mu}}\right)^{1-\delta} \frac{d t}{(1-t)^{1-\delta}(1-x t)^{\mu(1-\delta)}}
\end{aligned}
$$

Since $(1-s) /\left(1-s^{1+\mu}\right)$ is a positive, continuous function on $[0,1]$, the function $\left(\mathcal{K} f_{\mu}\right)(x)$ diverges to $+\infty$ as $x \rightarrow 1$ if $\mu \geq \frac{\delta}{1-\delta}$, and converges to a positive value then if $\mu<\frac{\delta}{1-\delta}$. Even in the case $\mu<\frac{\delta}{1-\delta}$, the function $\mathcal{K} f_{\mu}$ does not belong to $C^{1}[0,1]$ unless $\mu=0$. To see it, we note that

$$
\begin{aligned}
\left(\mathcal{K} f_{\mu}\right)^{\prime}(x) & =\frac{\delta-1}{x} \int_{0}^{1} \frac{\int_{t}^{1} s \frac{d}{d s} f_{\mu}(x s) d s}{\left(\int_{t}^{1} f_{\mu}(x s) d s\right)^{2-\delta}} d t \\
& =\frac{\delta-1}{x} \int_{0}^{1} \frac{f_{\mu}(x)-t f_{\mu}(x t)}{\left(\int_{t}^{1} f_{\mu}(x s) d s\right)^{2-\delta}} d t+\frac{1-\delta}{x}\left(\mathcal{K} f_{\mu}\right)(x)
\end{aligned}
$$

and that

$$
\begin{aligned}
& (1+\mu)^{\delta-2} \int_{0}^{1} \frac{f_{\mu}(x)-t f_{\mu}(x t)}{\left(\int_{t}^{1} f_{\mu}(x s) d s\right)^{2-\delta}} d t \\
& =\int_{0}^{1}\left(\frac{1-\left(\frac{1-x}{1-x t}\right)}{1-\left(\frac{1-x}{1-x t}\right)^{1+\mu}}\right)^{2-\delta} \frac{(1-x)^{\mu}-t(1-x t)^{\mu}}{(1-t)^{2-\delta}(1-x t)^{\mu(2-\delta)}} d t \\
& =-\int_{0}^{1}\left(\frac{1-\left(\frac{1-x}{1-x t}\right)}{1-\left(\frac{1-x}{1-x t}\right)^{1+\mu}}\right)^{2-\delta}\left(\frac{1-\left(\frac{1-x}{1-x t}\right)^{\mu}}{1-\left(\frac{1-x}{1-x t}\right)}\right) \frac{x}{(1-t)^{1-\delta}(1-x t)^{1+\mu(1-\delta)}} d t \\
& +\int_{0}^{1}\left(\frac{1-\left(\frac{1-x}{1-x t}\right)}{1-\left(\frac{1-x}{1-x t}\right)^{1+\mu}}\right)^{2-\delta} \frac{1}{(1-t)^{1-\delta}(1-x t)^{\mu(1-\delta)}} d t .
\end{aligned}
$$

The second term in the right-hand side of the above equality converges as $x \rightarrow 1$ provided that $\mu<\frac{\delta}{1-\delta}$, while the first term there diverges then because of $(1+$ $\mu)(1-\delta)>0$, unless $\mu=0$. Hence $\left(\mathcal{K} f_{\mu}\right)^{\prime}(x) \rightarrow \infty$ even though $\mu<\frac{\delta}{1-\delta}$ where $\mathcal{K} f_{\mu} \in C[0,1]$. Thus, in the case of $\mu>-1, \mu \neq 0$, the function $\left(\mathcal{K} f_{\mu}\right)(x)$ has the point $x=1$ as a singularity in the sense that $\mathcal{K} f_{\mu} \notin C^{1}[0,1]$. 
We shall next treat the case $\mu \leq-1$. Then we get

$$
\begin{aligned}
& \left(\mathcal{K} f_{\mu}\right)(x)=(-(1+\mu))^{1-\delta} \int_{0}^{1}\left(\frac{x}{(1-x)^{1+\mu}-(1-x t)^{1+\mu}}\right)^{1-\delta} d t \\
& \quad=(-(1+\mu))^{1-\delta}(1-x)^{-(1+\mu)(1-\delta)} \int_{0}^{1}\left(\frac{1-\left(\frac{1-x}{1-x t}\right)}{1-\left(\frac{1-x}{1-x t}\right)^{-(1+\mu)}}\right)^{1-\delta}\left(\frac{1-x t}{1-t}\right)^{1-\delta} d t,
\end{aligned}
$$

provided that $\mu \neq-1$. By $-(1+\mu)(1-\delta)>0$ this implies that $\left(\mathcal{K} f_{\mu}\right)(1)=0$. Also when $\mu=-1$ we can draw the same conclusion by an easy computation. Thus, in the case $\mu \leq-1$, the function $\left(\mathcal{K} f_{\mu}\right)(x)$ has the point $x=1$ as a singularity in the sense that $\left(\mathcal{K} f_{\mu}\right)(1)=0$. In this way we have shown that, for each $\mu \neq 0$, the function $f_{\mu}(x)=(1-x)^{\mu}$ is transformed by $\mathcal{K}$ to a function that has the point $x=1$ as a singularity in the sense that either $\mathcal{K} f_{\mu} \notin C^{1}[0,1]$ or $\left(\mathcal{K} f_{\mu}\right)(1)=0$. This situation is illustrated in Figure 4.2.
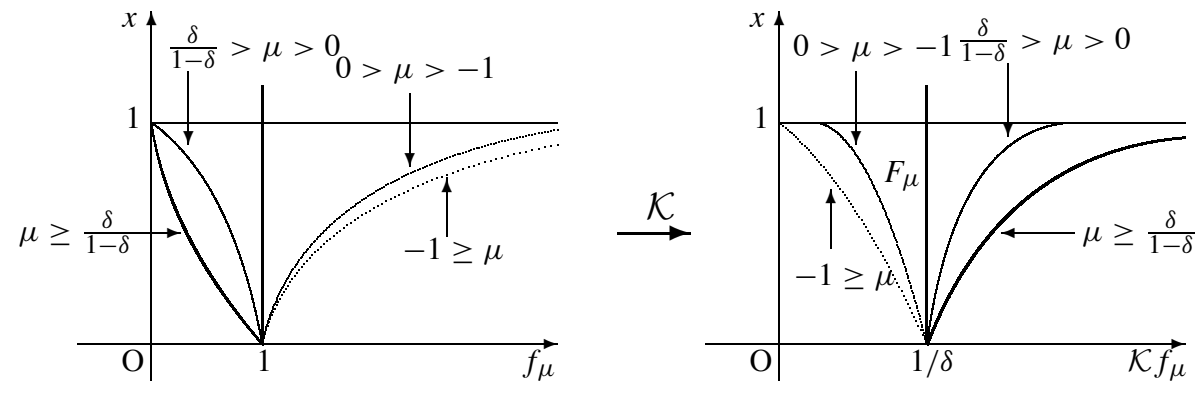

Figure 4.2. $(1-x)^{\mu}$ and $\mathcal{K}(1-x)^{\mu}$.

It is of worth to mention that Example 4.5 means that the transform $\mathcal{K}$ is not necessarily bijective from $\mathcal{M}^{0, \alpha}(I)_{\eta}$ onto $\mathcal{M}^{0, \alpha+\delta}(I)_{\eta}$. We focus on the case $\frac{\delta-1}{2-\delta}<\mu<\frac{\delta}{1-\delta}, \mu \neq 0$ and define $F_{\mu}:=\mathcal{K} f_{\mu}$ in this case. It follows from (4.8) that $F_{\mu}^{\prime}(x)$ can be estimated as

$$
F_{\mu}^{\prime}(x)=O\left(\int_{0}^{1} \frac{d t}{(1-t)^{1-\delta}(1-x t)^{1+\mu(1-\delta)}}\right)
$$

when $x \rightarrow 1$. Here $O$ stands for Landau's symbol. With the aid of the formula

$$
\int_{0}^{1} \frac{d t}{(1-t)^{1-\delta}(1-x t)^{1+\mu(1-\delta)}}=(1-x)^{-(1+\mu)(1-\delta)} \int_{0}^{1} \frac{d s}{s^{1-\delta}(1-x s)^{\delta-\mu(1-\delta)}}
$$

(a transformation formula on Gauss' hypergeometric function), which is verified by the substitution $t=(1-s) /(1-x s)$, we have

$$
F_{\mu}^{\prime}(x)=O\left((1-x)^{-(1+\mu)(1-\delta)}\right)
$$


as $x \rightarrow 1$. Noting that $-1<-(1+\mu)(1-\delta)<0$, we get the estimate

$$
\begin{aligned}
\left|F_{\mu}(x)-F_{\mu}(y)\right| & =\left|\int_{y}^{x}\right| F_{\mu}^{\prime}(\xi)|d \xi| \\
& \leq \exists M\left|(1-x)^{1-(1+\mu)(1-\delta)}-(1-y)^{1-(1+\mu)(1-\delta)}\right| \\
& \leq \exists M^{\prime}|x-y|^{1-(1+\mu)(1-\delta)}
\end{aligned}
$$

for $0 \leq x, y \leq 1$, where $M, M^{\prime}$ are constants independent of $x, y$. Let us now confine further ourselves to the case $\frac{\delta-1}{2-\delta}<\mu<0$. In this case we can take a number $\alpha$ so that $0<\alpha \leq(1-\delta)(-\mu)$ since $(1-\delta)(-\mu)>0$. Then $\alpha$ satisfies $\alpha+\delta \leq 1-(1+\mu)(1-\delta)<1$. Hence, by the above estimate, we see that $F_{\mu} \in \overline{\mathcal{M}}^{0, \alpha+\delta}[0,1]_{\eta}$ for each $\eta \in(0, \alpha]$. Thus if $\frac{\delta-1}{2-\delta}<\mu<0$, then, by taking $\alpha, \eta$ so that $0<\eta \leq \alpha \leq(1-\delta)(-\mu)$, the function $F_{\mu}$ belongs to $\mathcal{M}^{0, \alpha+\delta}[0,1]_{\eta}$. However there is not a function $f \in \mathcal{M}^{0, \alpha}[0,1]_{\eta}$ such that $\mathcal{K} f=F_{\mu}$, because, by Remark 4.4, the function $f_{\mu}(x)=(1-x)^{\mu}$ is the only solution in $\mathcal{M}^{0, \alpha}[0, B]_{\eta}$ of $\mathcal{K} f=F_{\mu}$ for each $B<1$, and so, if there were a solution $f \in \mathcal{M}^{0, \alpha}[0,1]_{\eta}$ then it would equal to $f_{\mu}(x)$ on $[0,1)$; it is impossible since $f_{\mu}(x) \rightarrow \infty$ as $x \rightarrow 1$ in view of $\mu<0$.

\section{Continuity}

In Proposition 4.3, we have established that the transform $\mathcal{K}: \mathcal{M}^{1, \alpha}(I)_{\eta} \rightarrow$ $\mathcal{M}^{1, \alpha+\delta}(I)_{\eta}$ is a bijection. In this section we shall show that the inverse $\mathcal{K}^{-1}$ : $\mathcal{M}^{1, \alpha+\delta}(I)_{\eta} \rightarrow \mathcal{M}^{1, \alpha}(I)_{\eta}$ is continuous. Throughout the section, let $f_{i} \in \mathcal{M}^{1, \alpha}(I)_{\eta}$, $i=1,2$, be solutions of $\mathcal{K} f_{i}=F_{i}$ for $F_{i} \in \mathcal{M}^{1, \alpha+\delta}(I)_{\eta}$ respectively. We first treat the case where $F_{1}(0)=F_{2}(0)=\gamma$, where $\gamma$ is a number defined in (2.1). This additional condition implies that $f_{1}(0)=f_{2}(0)=1$.

Lemma 5.1. Let $F_{1} \in \mathcal{M}^{1, \alpha+\delta}(I)_{\eta}, F_{1}(0)=\gamma$, and let $f_{1} \in \mathcal{M}^{1, \alpha}(I)_{\eta}$ satisfy $\mathcal{K} f_{1}=F_{1}$. Then there exist constants $\kappa_{0}$ and $M_{0}$ such that if $F_{2} \in \mathcal{M}^{1, \alpha+\delta}(I)$ satisfies $F_{2}(0)=F_{1}(0)$ then the solution $f_{2} \in \mathcal{M}^{1, \alpha}(I)_{\eta}$ of $\mathcal{K} f_{2}=F_{2}$ satisfies

$$
\left\|f_{2}^{\kappa}-f_{1}^{\kappa}\right\|_{1, \alpha, \eta ; \kappa} \leq M_{0}\left\|F_{2}-F_{1}\right\|_{1, \alpha+\delta, \eta} .
$$

for any $\kappa \leq \kappa_{0}$. Here $f_{i}^{\kappa}, i=1,2$, denote the sections of $f_{i}$ on $I_{\kappa}:=\{\kappa x: x \in I\}$, and $\|\cdot\|_{1, \alpha, \eta ; \kappa}$ denotes the norm in $\mathcal{C}^{1, \alpha}\left(I_{\kappa}\right)_{\eta}$.

Proof. By the assumption, $\mathcal{L}\left(f_{i}-1\right)=F_{i}-\gamma, i=1$, 2, on $I$. By Lemma $2.5, \mathcal{L}$ maps a neighborhood $U$ of 0 in $\mathcal{C}^{1, \alpha}(I)_{\eta}$ homeomorphically onto a neighborhood $V$ of 0 in $\mathcal{C}^{1, \alpha+\delta}(I)_{\eta}$. Since this map is a $C^{1}$-diffeomorphism, there is a constant $M_{0}$ independent of $G_{1}, G_{2}$ such that

$$
\left\|\mathcal{L}^{-1}\left(G_{2}\right)-\mathcal{L}^{-1}\left(G_{1}\right)\right\|_{1, \alpha, \eta} \leq M_{0}\left\|G_{2}-G_{1}\right\|_{1, \alpha+\delta, \eta}
$$

for $G_{1}, G_{2} \in V$. 
As in the proof of Proposition 2.1, let $\kappa>0$ and let $f_{i}^{\kappa}, F_{i}^{\kappa}$ be sections of $f_{i}$ and $F_{i}$ on $I_{\kappa}$. By $\left\|S_{\kappa}\left(F_{1}^{\kappa}-\gamma\right)\right\|_{1, \alpha+\delta, \eta}=\kappa^{\eta}\left\|F_{1}^{\kappa}-\gamma\right\|_{1, \alpha+\delta, \eta ; \kappa} \leq \kappa^{\eta} \| F_{1}-$ $\gamma \|_{1, \alpha+\delta, \eta}$, there exists a positive number $\kappa_{0}$ such that if $\kappa \leq \kappa_{0}$ then $S_{\kappa}\left(F_{1}^{\kappa}-\gamma\right)$ belongs to the neighborhood $V$ mentioned above. Moreover

$$
\begin{aligned}
\left\|S_{\kappa}\left(F_{2}^{\kappa}-\gamma\right)-S_{\kappa}\left(F_{1}^{\kappa}-\gamma\right)\right\|_{1, \alpha+\delta, \eta} & =\kappa^{\eta}\left\|F_{2}^{\kappa}-F_{1}^{\kappa}\right\|_{1, \alpha+\delta, \eta ; \kappa} \\
& \leq \kappa^{\eta}\left\|F_{2}-F_{1}\right\|_{1, \alpha+\delta, \eta} .
\end{aligned}
$$

Hence there exists a positive number $\rho$ such that if $\left\|F_{2}-F_{1}\right\|_{1, \alpha+\delta, \eta}<\rho$ then $S_{\kappa}\left(F_{2}^{\kappa}-\gamma\right)$ also belongs to $V$. Since $\mathcal{L}\left(S_{\kappa}\left(f_{i}^{\kappa}-1\right)\right)=S_{\kappa}\left(F_{i}^{\kappa}-\gamma\right), i=1,2$, and, in view of Proposition 4.3, the solution $g$ of $\mathcal{L}(g)=G$ is unique in $\mathcal{C}^{1, \alpha}(I)_{\eta}$ we see from Lemma 2.5 that $S_{\kappa}\left(f_{i}^{\kappa}-1\right)$ belong to $U$. Thus

$$
\mathcal{L}^{-1}\left(S_{\kappa}\left(F_{i}^{\kappa}-\gamma\right)\right)=S_{\kappa}\left(f_{i}^{\kappa}-1\right)
$$

by $\mathcal{L}^{-1}: V \rightarrow U$. By applying (5.1) to $G_{i}=S_{\kappa}\left(F_{i}^{\kappa}-\gamma\right)$, we get

$$
\left\|S_{\kappa}\left(f_{2}^{\kappa}-f_{1}^{\kappa}\right)\right\|_{1, \alpha, \eta} \leq M_{0}\left\|S_{\kappa}\left(F_{2}^{\kappa}-F_{1}^{\kappa}\right)\right\|_{1, \alpha+\delta, \eta} \leq M_{0} \kappa^{\eta}\left\|F_{2}-F_{1}\right\|_{1, \alpha+\delta, \eta} .
$$

Since $\left\|S_{\kappa}\left(f_{2}^{\kappa}-f_{1}^{\kappa}\right)\right\|_{1, \alpha, \eta}=\kappa^{\eta}\left\|f_{2}^{\kappa}-f_{1}^{\kappa}\right\|_{1, \alpha, \eta ; \kappa}$, we arrive at

$$
\left\|f_{2}^{\kappa}-f_{1}^{\kappa}\right\|_{1, \alpha, \eta ; \kappa} \leq M_{0}\left\|F_{2}-F_{1}\right\|_{1, \alpha+\delta, \eta} .
$$

This completes the proof.

Let $\mathcal{R}_{\kappa}$ be the restriction operation which assigns to each $f \in \mathcal{M}^{1, \alpha}(I)_{\eta}$ the restriction $\left.f\right|_{I_{\kappa}}$ on $I_{\kappa}$. Lemma 5.1 tells us that $\mathcal{R}_{\kappa} \mathcal{K}^{-1}: \mathcal{M}^{1, \alpha+\delta}(I)_{\eta} \rightarrow$ $\mathcal{M}^{1, \alpha}\left(I_{\kappa}\right)_{\eta}$ is continuous if $\kappa$ is sufficiently small. We turn our attention to the continuity of $\mathcal{R}_{\kappa}^{c} \mathcal{K}^{-1}$, where $\mathcal{R}_{\kappa}^{c}$ denotes a restriction operation which assigns to each $f \in \mathcal{M}^{1, \alpha}(I)_{\eta}$ the restriction of $f$ on the closure $\overline{I \backslash I_{\kappa}}$ of the complement of $I_{\kappa}$. It is enough to consider the case where $I=[0, B], \overline{I \backslash I_{\kappa}}=[\kappa B, B]$. We take $\kappa$ so that $0<\kappa<\kappa_{0}$ and set $b:=\kappa B$.

Lemma 5.2. The mapping

$$
\mathcal{R}_{\kappa}^{c} \mathcal{K}^{-1}:\left\{F \in \mathcal{M}^{1, \alpha+\delta}[0, B]_{\eta}: F(0)=\gamma\right\} \rightarrow C^{1}[b, B]
$$

is continuous. In particular, there are positive constants $\rho, M$ such that

$$
\left\|F_{2}-F_{1}\right\|_{1, \alpha+\delta, \eta}<\rho \Longrightarrow\left\|f_{2}-f_{1}\right\|_{C^{1}[b, B]} \leq M\left\|F_{2}-F_{1}\right\|_{1, \alpha+\delta, \eta} .
$$

Proof. We proceed in four steps.

Step 1 . Let $F_{1} \in \mathcal{M}^{1, \alpha+\delta}[0, B]_{\eta}$ be fixed and let $f_{1} \in \mathcal{M}^{1, \alpha}[0, B]_{\eta}$ be the solution of $\mathcal{K} f_{1}=F_{1}$. We may assume that $F_{1}(0)=\gamma$ and so $f_{1}(0)=1$. If $F_{2} \in \mathcal{M}^{1, \alpha+\delta}[0, B]_{\eta}$ satisfies $F_{2}(0)=\gamma$ and is sufficiently near $F_{1}$, say $\left\|F_{2}-F_{1}\right\|_{1, \alpha+\delta, \eta}<\varepsilon / M_{0}$ with 
$M_{0}$ in Lemma 5.1 and sufficiently small $\varepsilon$, then, by the lemma, the solution $f_{2}$ of $\mathcal{K} f_{2}=F_{2}$ satisfies $\max _{0 \leq x \leq b}\left|f_{2}(x)-f_{1}(x)\right|<\varepsilon$ and so $\min _{0 \leq x \leq b} f_{2}(x)>$ $\min _{0 \leq x \leq b} f_{1}(x)-\varepsilon$. Moreover an inspection to the proof of Lemma 3.6 shows that for any $M>0$ there exists $m>0$ such that if $\max _{b \leq x \leq B}|F(x)|+\left|F^{\prime}(x)\right| \leq M$ then a solution $f$ of $\mathcal{K} f=F$ satisfies $\min _{b \leq x \leq B} f(x) \geq m$. Hence there are positive numbers $\rho, m_{0}$ such that

$$
\text { if }\left\|F_{2}-F_{1}\right\|_{1, \alpha+\delta, \eta}<\rho \text { then } \min _{0 \leq x \leq B} f_{i}(x) \geq m_{0}, \quad i=1,2 \text {. }
$$

Step 2. Since $\mathcal{K} f=F$ is equivalent to (3.14), we obtain

$$
\begin{aligned}
& f_{2}(x)\left\{\frac{x^{\beta \delta} F_{2}(x)}{\left(\int_{0}^{x} r^{\beta-1} f_{2}(r) d r\right)^{\delta}}+\delta \int_{0}^{x} \frac{\xi^{\beta-1} x^{\beta \delta} F_{2}(x)-x^{\beta-1} \xi^{\beta \delta} F_{2}(\xi)}{\left(\int_{\xi}^{x} r^{\beta-1} f_{2}(r) d r\right)^{\delta+1}} f_{2}(\xi) d \xi\right\} \\
& =f_{1}(x)\left\{\frac{x^{\beta \delta} F_{1}(x)}{\left(\int_{0}^{x} r^{\beta-1} f_{1}(r) d r\right)^{\delta}}+\delta \int_{0}^{x} \frac{\xi^{\beta-1} x^{\beta \delta} F_{1}(x)-x^{\beta-1} \xi^{\beta \delta} F_{1}(\xi)}{\left(\int_{\xi}^{x} r^{\beta-1} f_{1}(r) d r\right)^{\delta+1}} f_{1}(\xi) d \xi\right\} .
\end{aligned}
$$

By setting $(\delta F)(x):=F_{1}(x)-F_{2}(x), f(\tau, x):=(1-\tau) f_{1}(x)+\tau f_{2}(x)$, this is rewritten as

$$
\begin{aligned}
& \left(f_{2}(x)-f_{1}(x)\right)\left\{\frac{x^{\beta \delta} F_{2}(x)}{\left(\int_{0}^{x} r^{\beta-1} f_{2}(r) d r\right)^{\delta}}\right. \\
& \left.+\delta \int_{0}^{x} \frac{\xi^{\beta-1} x^{\beta \delta} F_{2}(x)-x^{\beta-1} \xi^{\beta \delta} F_{2}(\xi)}{\left(\int_{\xi}^{x} r^{\beta-1} f_{2}(r) d r\right)^{\delta+1}} f_{2}(\xi) d \xi\right\} \\
& =f_{1}(x)\left\{\frac{x^{\beta \delta}(\delta F)(x)}{\left(\int_{0}^{x} r^{\beta-1} f_{1}(r) d r\right)^{\delta}}\right. \\
& \left.+\delta \int_{0}^{x} \frac{\xi^{\beta-1} x^{\beta \delta}(\delta F)(x)-x^{\beta-1} \xi^{\beta \delta}(\delta F)(\xi)}{\left(\int_{\xi}^{x} r^{\beta-1} f_{1}(r) d r\right)^{\delta+1}} f_{1}(\xi) d \xi\right\} \\
& -f_{1}(x)\left\{\int_{0}^{1} \frac{d}{d \tau} \frac{x^{\beta \delta} F_{2}(x)}{\left(\int_{0}^{x} r^{\beta-1} f(\tau, r) d r\right)^{\delta}} d \tau\right. \\
& \left.+\delta \int_{0}^{1} \frac{d}{d \tau} \int_{0}^{x} \frac{\xi^{\beta-1} x^{\beta \delta} F_{2}(x)-x^{\beta-1} \xi^{\beta \delta} F_{2}(\xi)}{\left(\int_{\xi}^{x} r^{\beta-1} f(\tau, r) d r\right)^{\delta+1}} f(\tau, \xi) d \xi d \tau\right\} .
\end{aligned}
$$


Hence, by operations similar to those carried out for the deduction of (4.6), we have

$$
f_{2}(x)-f_{1}(x)=R(x)+\int_{0}^{x} \Gamma(x, \xi)\left(f_{2}(\xi)-f_{1}(\xi)\right) d \xi, \quad 0 \leq x \leq B,
$$

where we define a function $R(x)$ and a function $\Gamma(x, \xi)$ on $0 \leq \xi<x \leq B$ by

$$
\begin{aligned}
R(x)= & f_{1}(x)\left\{\frac{x^{\beta \delta}(\delta F)(x)}{\left(\int_{0}^{x} r^{\beta-1} f_{1}(r) d r\right)^{\delta}}\right. \\
& \left.+\delta \int_{0}^{x} \frac{\xi^{\beta-1} x^{\beta \delta}(\delta F)(x)-x^{\beta-1} \xi^{\beta \delta}(\delta F)(\xi)}{\left(\int_{\xi}^{x} r^{\beta-1} f_{1}(r) d r\right)^{\delta+1}} f_{1}(\xi) d \xi\right\}
\end{aligned}
$$

and

$$
\begin{aligned}
\Gamma(x, \xi): & =\delta \frac{\sin \pi \delta}{\pi} f_{1}(x) f_{2}(x) \times \\
& \left\{\int_{0}^{1} \frac{\xi^{\beta-1} x^{\beta \delta} F_{2}(x)}{\left(\int_{0}^{x} r^{\beta-1} f(\tau, r) d r\right)^{\delta+1}} d \tau-\int_{0}^{1} \frac{\xi^{\beta-1} x^{\beta \delta} F_{2}(x)-x^{\beta-1} \xi^{\beta \delta} F_{2}(\xi)}{\left(\int_{\xi}^{x} r^{\beta-1} f(\tau, r) d r\right)^{\delta+1}} d \tau\right. \\
& \left.+(\delta+1) \xi^{\beta-1} \int_{0}^{1} d \tau \int_{0}^{\xi} \frac{s^{\beta-1} x^{\beta \delta} F_{2}(x)-x^{\beta-1} s^{\beta \delta} F_{2}(s)}{\left(\int_{s}^{x} r^{\beta-1} f(\tau, r) d r\right)^{\delta+2}} f(\tau, s) d s\right\} .
\end{aligned}
$$

Step 3. We suppose that $\left\|F_{2}-F_{1}\right\|_{1, \alpha+\delta, \eta}<\rho$. Then, by (5.2), we get

$$
\begin{aligned}
& \left|\frac{\xi^{\beta-1} x^{\beta \delta} F_{2}(x)-x^{\beta-1} \xi^{\beta \delta} F_{2}(\xi)}{\left(\int_{\xi}^{x} r^{\beta-1} f(\tau, r) d r\right)^{\delta+1}}\right| \leq \frac{\beta^{\delta+1}}{m_{0}^{\delta+1}} \xi^{\beta-1} x^{\beta-1} \frac{\int_{\xi}^{x}\left|\left(r^{1-\beta+\beta \delta} F_{2}(r)\right)^{\prime}\right| d r}{\left(x^{\beta}-\xi^{\beta}\right)^{\delta+1}} \\
& \leq \frac{\beta^{\delta+1}}{m_{0}^{\delta+1} \xi^{\beta-1} x^{\beta-1}} \frac{\xi^{-\beta+\beta \delta}(x-\xi)\left(\left|F_{2}\right|_{0}+\left|\theta F_{2}\right|_{0}\right)}{\left(x^{\beta}-\xi^{\beta}\right)^{\delta+1}} \leq M_{0} \frac{1}{\xi}\left(\frac{\xi}{x}\right)^{\beta \delta} \frac{x^{\delta}}{(x-\xi)^{\delta}},
\end{aligned}
$$

where $M_{0}$ is a constant independent of $F_{2}, f_{2}$. This leads to the estimates

$$
\begin{gathered}
|\Gamma(x, \xi)| \leq M_{\Gamma} f_{2}(x) \frac{1}{\xi}\left(\frac{\xi}{x}\right)^{\beta \delta} \frac{x^{\delta}}{(x-\xi)^{\delta}}, \quad 0<\xi<x \leq B, \\
|R(x)| \leq M_{R}\|\delta F\|_{1, \alpha+\delta, \eta}=M_{R}\left\|F_{2}-F_{1}\right\|_{1, \alpha+\delta, \eta},
\end{gathered}
$$

where $M_{\Gamma}, M_{R}$ are constants independent of $F_{2}, f_{2}$.

We set $\Gamma^{0}(x, \xi):=\Gamma(x, \xi) / f_{2}(x)$ and

$$
R_{1}(x):=R(x)+f_{1}(x) \int_{0}^{b} \Gamma^{0}(x, \xi)\left(f_{2}(\xi)-f_{1}(\xi)\right) d \xi
$$


Then, for $b \leq x \leq B$, equation (5.3) is recast as

$$
\begin{aligned}
& \left(1-\int_{0}^{x} \Gamma^{0}(x, \xi)\left(f_{2}(\xi)-f_{1}(\xi)\right) d \xi\right)\left(f_{2}(x)-f_{1}(x)\right) \\
& =R_{1}(x)+f_{1}(x) \int_{b}^{x} \Gamma^{0}(x, \xi)\left(f_{2}(\xi)-f_{1}(\xi)\right) d \xi, \quad b \leq x \leq B .
\end{aligned}
$$

By (5.4) we have $\left|\Gamma^{0}(x, \xi)\right| \leq M_{\Gamma} \xi^{\beta \delta-1} x^{-\beta \delta}(1-(\xi / x))^{-\delta}$. Accordingly,

$d(x):=\left|\int_{0}^{x} \Gamma^{0}(x, \xi)\left(f_{2}(\xi)-f_{1}(\xi)\right) d \xi\right| \leq M_{\Gamma} B(\beta \delta, 1-\delta) \max _{0 \leq \xi \leq x}\left|f_{2}(\xi)-f_{1}(\xi)\right|$

for $b \leq x \leq B$. By Lemma 5.1, if $\left\|F_{2}-F_{1}\right\|_{1, \alpha+\delta, \eta}$ is sufficiently small, then $d(b) \leq \frac{1}{4}$, and so, if $c>b$ is enough near $b$ then $d(c) \leq \frac{1}{2}$ and hence

$$
\left(1-\int_{0}^{x} \Gamma^{0}(x, \xi)\left(f_{2}(\xi)-f_{1}(\xi)\right) d \xi\right)^{-1} \leq 2
$$

on the interval $[b, c]$. Similarly, it follows from (5.4), (5.5), Lemma 5.1 that there exists a constant $M_{R}^{b}$ such that $\left|R_{1}(x)\right| \leq 2^{-1} M_{R}^{b}\left\|F_{2}-F_{1}\right\|_{1, \alpha+\delta, \eta}$ for $b \leq x \leq B$. Further, by (5.4), we get the estimate $\left|\Gamma^{0}(x, \xi)\right| \leq 2^{-1} M_{\Gamma}^{b}(x-\xi)^{-\delta}$ for $b \leq \xi<$ $x \leq B$. In this way, from (5.6), we have the integral inequality

$$
\left|f_{2}(x)-f_{1}(x)\right| \leq M_{R}^{b}\left\|F_{2}-F_{1}\right\|_{1, \alpha+\delta, \eta}+M_{\Gamma}^{b} f_{1}(x) \int_{b}^{x} \frac{1}{(x-\xi)^{\delta}}\left|f_{2}(\xi)-f_{1}(\xi)\right| d \xi
$$

for $b \leq x \leq c$. Here the constants $M_{R}^{b}$ and $M_{\Gamma}^{b}$ are independent of $F_{2}, f_{2}$. By the Gronwall inequality, this inequality is solved as

$$
\left|f_{2}(x)-f_{1}(x)\right| \leq M_{R}^{b}\left\|F_{2}-F_{1}\right\|_{1, \alpha+\delta, \eta}+L \int_{b}^{x} \frac{1}{(x-\xi)^{\delta}} M_{R}^{b}\left\|F_{2}-F_{1}\right\|_{1, \alpha+\delta, \eta} d \xi
$$

for $b \leq x \leq c$ with a constant $L$ independent of $F_{2}, f_{2}$. Hence, if $\left\|F_{2}-F_{1}\right\|_{1, \alpha+\delta, \eta}$ is sufficiently small then $\max _{b \leq x \leq c}\left|f_{2}(x)-f_{1}(x)\right|$ is enough small so that

$$
\left|\int_{b}^{c} \Gamma^{0}(c, \xi)\left(f_{2}(\xi)-f_{1}(\xi)\right) d \xi\right| \leq \frac{1}{8}
$$

as long as $d(c) \leq \frac{1}{2}$. This shows that (5.7) still holds even at $x=B$, because if $d(x)>\frac{1}{2}$ for some $x \in[b, B]$ then, at a point $x^{*}$ for which $d\left(x^{*}\right)=\frac{1}{2}$, we would have the contradiction

$$
\begin{aligned}
d\left(x^{*}\right) & =\left|\int_{0}^{b} \Gamma^{0}\left(x^{*}, \xi\right)\left(f_{2}(\xi)-f_{1}(\xi)\right) d \xi+\int_{b}^{x^{*}} \Gamma^{0}\left(x^{*}, \xi\right)\left(f_{2}(\xi)-f_{1}(\xi)\right) d \xi\right| \\
& \leq \frac{1}{4}+\frac{1}{8}<\frac{1}{2} .
\end{aligned}
$$


Hence we conclude that there is a constant $M_{B}^{0}$ such that

$$
\left|f_{2}(x)-f_{1}(x)\right| \leq M_{B}^{0}\left\|F_{2}-F_{1}\right\|_{1, \alpha+\delta, \eta}
$$

for $b \leq x \leq B$.

Step 4. We set $\Gamma_{1}(x, \xi):=\int_{\xi}^{x} \Gamma(x, z) d z=f_{2}(x) \int_{\xi}^{x} \Gamma^{0}(x, z) d z$. Then, by (5.4), we have the estimate $\left|\Gamma_{1}(x, \xi)\right| \leq M(1-(\xi / x))^{1-\delta}$ with a constant $M$. Hence an integration by parts to (5.3) shows that

$$
f_{2}(x)-f_{1}(x)=R(x)-\int_{0}^{x} \Gamma_{1}(x, \xi)\left(f_{2}^{\prime}(\xi)-f_{1}^{\prime}(\xi)\right) d \xi .
$$

Here we used $f_{2}(0)=f_{1}(0)$ that follows from the assumption $F_{2}(0)=F_{1}(0)$. By a similar computation to that in (4.4), it is verified that $\left|\frac{\partial}{\partial x} \Gamma_{1}(x, \xi)\right| \leq M_{1}(x-\xi)^{-\delta}$ for $b \leq x \leq B, 0 \leq \xi<x \leq B$ with a constant $M_{1}$. Hence, differentiating (5.8) and setting $\Gamma_{2}(x, \xi):=\frac{\partial}{\partial x} \Gamma_{1}(x, \xi)$, we get

$$
f_{2}^{\prime}(x)-f_{1}^{\prime}(x)=R^{\prime}(x)-\int_{0}^{x} \Gamma_{2}(x, \xi)\left(f_{2}^{\prime}(\xi)-f_{1}^{\prime}(\xi)\right) d \xi, \quad b \leq x \leq B .
$$

It is easy to see that $R^{\prime}(x) \in C[b, B]$ satisfies $\left|R^{\prime}(x)\right| \leq M_{R^{\prime}}\left\|F_{2}-F_{1}\right\|_{1, \alpha+\delta, \eta}$ with a constant $M_{R^{\prime}}$ independent of $F_{2}, f_{2}$. By the decomposition $-\Gamma_{2}(x, \xi)=$ $f_{2}^{\prime}(x) \Gamma_{21}(x, \xi)+\Gamma_{22}(x, \xi)$ with $\Gamma_{21}(x, \xi)=-\Gamma_{1}(x, \xi) / f_{2}(x)$ and $\Gamma_{22}(x, \xi)=$ $-f_{2}(x) \frac{\partial}{\partial x} \int_{\xi}^{x} \Gamma^{0}(x, z) d z$, this is written as

$$
\begin{aligned}
f_{2}^{\prime}(x)-f_{1}^{\prime}(x)= & R^{\prime}(x)+f_{2}^{\prime}(x) \int_{0}^{x} \Gamma_{21}(x, \xi)\left(f_{2}^{\prime}(\xi)-f_{1}^{\prime}(\xi)\right) d \xi \\
& +\int_{0}^{x} \Gamma_{22}(x, \xi)\left(f_{2}^{\prime}(\xi)-f_{1}^{\prime}(\xi)\right) d \xi .
\end{aligned}
$$

From this, in a similar manner to that for the derivation of (5.6), we have

$$
\begin{aligned}
& \left(1-\int_{0}^{x} \Gamma_{21}(x, \xi)\left(f_{2}^{\prime}(\xi)-f_{1}^{\prime}(\xi)\right) d \xi\right)\left(f_{2}^{\prime}(x)-f_{1}^{\prime}(x)\right) \\
& =R_{2}(x)+\int_{b}^{x} \Gamma^{1}(x, \xi)\left(f_{2}^{\prime}(\xi)-f_{1}^{\prime}(\xi)\right) d \xi, \quad b \leq x \leq B,
\end{aligned}
$$

where $R_{2}(x)$ satisfies $\left|R_{2}(x)\right| \leq M_{R_{2}}^{b}\left\|F_{2}-F_{1}\right\|_{1, \alpha+\delta, \eta}$ and $\Gamma^{1}(x, \xi)$ satisfies $\left|\Gamma^{1}(x, \xi)\right| \leq M_{\Gamma^{1}}^{b}(x-\xi)^{-\delta}$ for $b \leq x \leq B, 0 \leq \xi<x \leq B$ with constants $M_{R_{2}}^{b}, M_{\Gamma^{1}}^{b}$ independent of $F_{2}, f_{2}$. Hence, as in Step 3, we conclude that there is a constant $M_{B}^{1}$ such that

$$
\left|f_{2}^{\prime}(x)-f_{1}^{\prime}(x)\right| \leq M_{B}^{1}\left\|F_{2}-F_{1}\right\|_{1, \alpha+\delta, \eta}
$$

for $b \leq x \leq B$. 
Our next task is to prove the following:

\section{Lemma 5.3. The inverse transform}

$$
\mathcal{K}^{-1}:\left\{F \in \mathcal{M}^{1, \alpha+\delta}[0, B]_{\eta}: F(0)=\gamma\right\} \rightarrow\left\{f \in \mathcal{M}^{1, \alpha}(I)_{\eta}: f(0)=1\right\}
$$

is continuous.

Proof. Assume that $F_{1}(0)=F_{2}(0)=\gamma$ and let $f_{i} \in \mathcal{M}^{1, \alpha}[0, B]_{\eta}, i=1,2$, be solutions of $\mathcal{K} f_{i}=F_{i}$ for $F_{i} \in \mathcal{M}^{1, \alpha+\delta}[0, B]_{\eta}$ respectively. Then, by (3.5),

$$
\begin{aligned}
& I_{0, w_{1}}^{\delta}\left(\frac{1}{x^{\beta-1} f_{2}(x)}-\frac{1}{x^{\beta-1} f_{1}(x)}\right) \\
& =\frac{1}{\Gamma(\delta)} x^{1-\beta+\beta \delta}\left(F_{2}(x)-F_{1}(x)\right)-\left(I_{0, w_{2}}^{\delta}-I_{0, w_{1}}^{\delta}\right) \frac{1}{x^{\beta-1} f_{2}(x)} .
\end{aligned}
$$

By setting $\rho=\frac{\eta+1}{\beta}-1$ in the commutative diagram (3.10), it follows that $I_{0, w_{1}}^{\delta}$ is a homeomorphism of $\mathcal{C}^{1, \alpha}[0, B]_{\eta+1-\beta}$ onto $\mathcal{C}^{1, \alpha+\delta}[0, B]_{\eta+1-\beta+\beta \delta}$ and the inverse is given by $D_{0, w_{1}}^{\delta}$.

The function $x^{1-\beta+\beta \delta}\left(F_{2}(x)-F_{1}(x)\right)$ belongs to $\mathcal{C}^{1, \alpha+\delta}[0, B]_{\eta+1-\beta+\beta \delta}$. Since the multiplication operator by $x^{1-\beta+\beta \delta}$ is a homeomorphism of $\mathcal{C}^{1, \alpha+\delta}[0, B]_{\eta}$ onto $\mathcal{C}^{1, \alpha+\delta}[0, B]_{\eta+1-\beta+\beta \delta}$,

$$
\left\|x^{1-\beta+\beta \delta}\left(F_{2}(x)-F_{1}(x)\right)\right\|_{1, \alpha+\delta, \eta+1-\beta+\beta \delta} \leq C_{1}\left\|F_{2}-F_{1}\right\|_{1, \alpha+\delta, \eta},
$$

where $C_{1}$ is a constant. On the other hand, by Lemma 5.1, if $\kappa \leq \kappa_{0}$ then $\| f_{2}-$ $f_{1}\left\|_{1, \alpha, \eta ; \kappa} \leq M_{0}\right\| F_{2}-F_{1} \|_{1, \alpha+\delta, \eta}$ and so, by (5.2),

$$
\left\|\frac{1}{x^{\beta-1} f_{2}(x)}-\frac{1}{x^{\beta-1} f_{1}(x)}\right\|_{1, \alpha, \eta+1-\beta ; \kappa} \leq C_{2}\left\|F_{2}-F_{1}\right\|_{1, \alpha+\delta, \eta},
$$

where $C_{2}$ is a constant. Hence, by (5.9),

$$
\left\|\left(I_{0, w_{2}}^{\delta}-I_{0, w_{1}}^{\delta}\right) \frac{1}{x^{\beta-1} f_{2}(x)}\right\|_{1, \alpha+\delta, \eta+1-\beta+\beta \delta ; \kappa} \leq C_{3}\left\|F_{2}-F_{1}\right\|_{1, \alpha+\delta, \eta}
$$

with a constant $C_{3}$. In other words, the second term in the right-hand side of (5.9) is small enough in $\mathcal{C}^{1, \alpha+\delta}[0, \kappa B]_{\eta+1-\beta+\beta \delta}$, provided that $F_{2}-F_{1}$ is sufficiently small in $\mathcal{C}^{1, \alpha+\delta}[0, B]_{\eta}$ and $0<\kappa \leq \kappa_{0}$.

Let $b<\kappa_{0} B$ be fixed and consider the function

$$
\begin{aligned}
p(x): & =\Gamma(\delta)\left(I_{0, w_{2}}^{\delta}-I_{0, w_{1}}^{\delta}\right) \frac{1}{x^{\beta-1} f_{2}(x)} \\
& =\int_{0}^{x}\left(\frac{\xi^{\beta-1} f_{2}(\xi)}{\left(\int_{\xi}^{x} r^{\beta-1} f_{2}(r) d r\right)^{1-\delta}}-\frac{\xi^{\beta-1} f_{1}(\xi)}{\left(\int_{\xi}^{x} r^{\beta-1} f_{1}(r) d r\right)^{1-\delta}}\right) \frac{d \xi}{\xi^{\beta-1} f_{2}(\xi)}
\end{aligned}
$$


on the interval $[b, B]$. We take $a$ so that $0<a<b$ and, using an integration by parts, we decompose $p(x)$ as $p(x)=p_{1}(x)+p_{2}(x)+p_{3}(x)$ where

$$
\begin{aligned}
& p_{1}(x):=\int_{0}^{a}\left\{\frac{\xi^{\beta-1} f_{2}(\xi)}{\left(\int_{\xi}^{x} r^{\beta-1} f_{2}(r) d r\right)^{1-\delta}}-\frac{\xi^{\beta-1} f_{1}(\xi)}{\left(\int_{\xi}^{x} r^{\beta-1} f_{1}(r) d r\right)^{1-\delta}}\right\} \frac{d \xi}{\xi^{\beta-1} f_{2}(\xi)}, \\
& p_{2}(x):=\frac{1}{\delta}\left\{\left(\int_{a}^{x} r^{\beta-1} f_{2}(r) d r\right)^{\delta}-\left(\int_{a}^{x} r^{\beta-1} f_{1}(r) d r\right)^{\delta}\right\} \frac{1}{a^{\beta-1} f_{2}(a)}, \\
& p_{3}(x):=\frac{1}{\delta} \int_{a}^{x}\left\{\left(\int_{\xi}^{x} r^{\beta-1} f_{2}(r) d r\right)^{\delta}-\left(\int_{\xi}^{x} r^{\beta-1} f_{1}(r) d r\right)^{\delta}\right\}\left(\frac{d}{d \xi} \frac{1}{\xi^{\beta-1} f_{2}(\xi)}\right) d \xi .
\end{aligned}
$$

It is easily seen from (5.2) that $p_{1}(x)$ is twice differentiable and

$$
\left|p_{1}^{\prime \prime}(x)\right| \leq \text { const. }\left\{\left\|f_{2}-f_{1}\right\|_{C^{1}[b, B]}+\left\|f_{2}^{\kappa}-f_{1}^{\kappa}\right\|_{1, \alpha, \eta ; \kappa}\right\}
$$

for $x \in[b, B]$. This, together with Lemmas 5.1 and 5.2, leads to

$$
\left|p_{1}^{\prime \prime}(x)\right| \leq \text { const. }\left\|F_{2}-F_{1}\right\|_{1, \alpha+\delta, \eta}
$$

for $x \in[b, B]$. Hence we have

$$
\left\|p_{1}(x)\right\|_{C^{1, \alpha}[b, B]} \leq \text { const. }\left\|F_{2}-F_{1}\right\|_{1, \alpha+\delta, \eta},
$$

where $\|\cdot\|_{C^{1, \alpha}[b, B]}$ is the standard norm of the Hölder space $C^{1, \alpha}[b, B]$. Similarly we have

$$
\left\|p_{2}(x)\right\|_{C^{1, \alpha}[b, B]} \leq \text { const. }\left\|F_{2}-F_{1}\right\|_{1, \alpha+\delta, \eta} .
$$

A computation shows that

$$
\begin{aligned}
p_{3}^{\prime}(x)= & \int_{a}^{x}\left\{\frac{x^{\beta-1} f_{2}(x)}{\left(\int_{\xi}^{x} r^{\beta-1} f_{2}(r) d r\right)^{1-\delta}}-\frac{x^{\beta-1} f_{1}(x)}{\left(\int_{\xi}^{x} r^{\beta-1} f_{1}(r) d r\right)^{1-\delta}}\right\} \times \\
& \left(\frac{d}{d \xi} \frac{1}{\xi^{\beta-1} f_{2}(\xi)}\right) d \xi \\
= & x^{\beta-1}\left(f_{2}(x)-f_{1}(x)\right) \int_{a}^{x} \frac{1}{\left(\int_{\xi}^{x} r^{\beta-1} f_{1}(r) d r\right)^{1-\delta}}\left(\frac{d}{d \xi} \frac{1}{\xi^{\beta-1} f_{2}(\xi)}\right) d \xi \\
& +x^{\beta-1} f_{2}(x) \int_{a}^{x}\left\{\frac{1}{\left(\int_{\xi}^{x} r^{\beta-1} f_{2}(r) d r\right)^{1-\delta}}-\frac{1}{\left(\int_{\xi}^{x} r^{\beta-1} f_{1}(r) d r\right)^{1-\delta}}\right\} \times \\
& \left(\frac{d}{d \xi} \frac{1}{\xi^{\beta-1} f_{2}(\xi)}\right) d \xi .
\end{aligned}
$$


Hence, by setting

$$
\begin{aligned}
& q_{1}(x):=\int_{a}^{x} \frac{1}{\left(\int_{\xi}^{x} r^{\beta-1} f_{1}(r) d r\right)^{1-\delta}}\left(\frac{d}{d \xi} \frac{1}{\xi^{\beta-1} f_{2}(\xi)}\right) d \xi \\
& q_{2}(x):=\int_{a}^{x}\left(\int_{0}^{1} \frac{\int_{\xi}^{x} r^{\beta-1}\left(f_{2}(r)-f_{1}(r)\right) d r}{\left(\int_{\xi}^{x} r^{\beta-1} f(\tau, r) d r\right)^{2-\delta}} d \tau\right)\left(\frac{d}{d \xi} \frac{1}{\xi^{\beta-1} f_{2}(\xi)}\right) d \xi,
\end{aligned}
$$

we have

$$
p_{3}{ }^{\prime}(x)=x^{\beta-1}\left(f_{2}(x)-f_{1}(x)\right) q_{1}(x)+(\delta-1) x^{\beta-1} f_{2}(x) q_{2}(x) .
$$

Set $\phi(\xi):=\frac{d}{d \xi} \frac{1}{\xi^{\beta-1} f_{2}(\xi)}$, which belongs to $C^{0, \alpha}[b, B]$, and let $b \leq y<x \leq B$. Then, as in [12, p.488], we decompose $q_{1}(x)-q_{1}(y)$ as

$$
\begin{aligned}
& q_{1}(x)-q_{1}(y) \\
& =\int_{a}^{x} \frac{\phi(\xi)}{\left(\int_{\xi}^{x} r^{\beta-1} f_{1}(r) d r\right)^{1-\delta}} d \xi-\int_{a}^{y} \frac{\phi(\xi)}{\left(\int_{\xi}^{y} r^{\beta-1} f_{1}(r) d r\right)^{1-\delta}} d \xi \\
& =\int_{y}^{x} \frac{\phi(\xi)-\phi(y)}{\left(\int_{\xi}^{x} r^{\beta-1} f_{1}(r) d r\right)^{1-\delta}} d \xi \\
& +\int_{a}^{y}\left\{\frac{1}{\left(\int_{\xi}^{x} r^{\beta-1} f_{1}(r) d r\right)^{1-\delta}}-\frac{1}{\left(\int_{\xi}^{y} r^{\beta-1} f_{1}(r) d r\right)^{1-\delta}}\right\}(\phi(\xi)-\phi(y)) d \xi \\
& \\
& +\left\{\int_{a}^{x} \frac{d \xi}{\left(\int_{\xi}^{x} r^{\beta-1} f_{1}(r) d r\right)^{1-\delta}}-\int_{a}^{y} \frac{d \xi}{\left(\int_{\xi}^{y} r^{\beta-1} f_{1}(r) d r\right)^{1-\delta}}\right\} \phi(y) . \\
& =: I_{1}+I_{2}+I_{3} .
\end{aligned}
$$

The first term $I_{1}$ of the right-hand side is estimated as

$$
\left|I_{1}\right| \leq\|\phi\|_{C^{0, \alpha}[b, B]} M \int_{y}^{x} \frac{(\xi-y)^{\alpha}}{(x-\xi)^{1-\delta}} d \xi=M_{1}\|\phi\|_{C^{0, \alpha}[b, B]}(x-y)^{\alpha+\delta},
$$

where $\|\phi\|_{C^{0, \alpha}[b, B]}$ denotes the standard norm of the Hölder space $C^{0, \alpha}[b, B]$, namely the norm with $w(x)=x$ in Lemma 3.3.(4), $M$ depends only on $f_{1}, \beta$, and $M_{1}=M B(\alpha+1, \delta)$. By the substitutions $\xi=y t, r=y s$, the second term $I_{2}$ of the right-hand side of (5.14) is written as

$$
\begin{aligned}
I_{2} & =\int_{a / y}^{1}(\ell(x / y, t)-\ell(1, t))(\phi(y t)-\phi(y)) y d t \\
& =\int_{a / y}^{1}(\phi(y t)-\phi(y)) y d t \int_{1}^{x / y} \frac{\partial \ell}{\partial u}(u, t) d u,
\end{aligned}
$$


where $\ell(u, t)=\left(\int_{t}^{u} y^{\beta} s^{\beta-1} f_{1}(y s) d s\right)^{\delta-1}$. Since

$$
\left|\frac{\partial \ell}{\partial u}(u, t)\right| \leq M(u-t)^{\delta-2}, \quad \frac{a}{B} \leq \frac{a}{y} \leq t<u \leq \frac{B}{b},
$$

with a constant $M$ independent of $y, t, u$, the term $I_{2}$ is estimated as

$$
\begin{aligned}
\left|I_{2}\right| & \leq M\|\phi\|_{C^{0, \alpha}[b, B]} \int_{a / y}^{1}(y-y t)^{\alpha} y d t \int_{1}^{x / y}(u-t)^{\delta-2} d u \\
& =M\|\phi\|_{C^{0, \alpha}[b, B]} \int_{a / y}^{1}(1-t)^{\alpha}\left\{(1-t)^{\delta-1}-\left(\frac{x}{y}-t\right)^{\delta-1}\right\} d t .
\end{aligned}
$$

But, by the L'Hôpital rule and the substitution $t=(1-s) /(1-(y / x) s)$, we have

$$
\begin{aligned}
& \lim _{\frac{x}{y} \rightarrow 1} \frac{1}{\left(\frac{x}{y}-1\right)^{\alpha+\delta}} \int_{0}^{1}(1-t)^{\alpha}\left\{(1-t)^{\delta-1}-\left(\frac{x}{y}-t\right)^{\delta-1}\right\} d t \\
& =\lim _{\frac{x}{y} \rightarrow 1} \frac{1-\delta}{\alpha+\delta} \frac{1}{\left(\frac{x}{y}-1\right)^{\alpha+\delta-1}} \int_{0}^{1}(1-t)^{\alpha}\left(\frac{x}{y}-t\right)^{\delta-2} d t \\
& =\frac{1-\delta}{\alpha+\delta} \lim _{\frac{x}{y} \rightarrow 1}\left(\frac{y}{x}\right)^{\alpha+1} \int_{0}^{1} \frac{s^{\alpha}}{\left(1-\frac{y}{x} s\right)^{\alpha+\delta}} d s=\frac{1-\delta}{\alpha+\delta} \int_{0}^{1} \frac{s^{\alpha}}{(1-s)^{\alpha+\delta}} d s<\infty .
\end{aligned}
$$

Hence, $\left|I_{2}\right| \leq M_{2}\|\phi\|_{C^{0, \alpha}[b, B]}(x-y)^{\alpha+\delta}$ for $b \leq y \leq x \leq B$ with a constant $M_{2}$ independent of $x, y$. By the substitution $\xi=x t, \xi=y t$, the third term $I_{3}$ of the right-hand side of (5.14) is written as

$$
\begin{aligned}
I_{3}= & x^{1-\beta(1-\delta)} \int_{a / x}^{a / y} \frac{d t}{\left(\int_{t}^{1} s^{\beta-1} f_{1}(x s) d s\right)^{1-\delta}} \phi(y) \\
& +\int_{a / y}^{1} d t \int_{y}^{x} \frac{d}{d z} \frac{z^{1-\beta(1-\delta)}}{\left(\int_{t}^{1} s^{\beta-1} f_{1}(z s) d s\right)^{1-\delta}} d z \phi(y) .
\end{aligned}
$$

Since $\left|(1-(a / x))^{\delta}-(1-(a / y))^{\delta}\right| \leq \exists M|x-y|$ for $b \leq y \leq x \leq B$ in view of $a<b$, the term $I_{3}$ is estimated as $\left|I_{3}\right| \leq M_{3}\|\phi\|_{C^{0, \alpha}[b, B]}(x-y)^{\alpha+\delta}$ for $b \leq y<$ $x \leq B$ with a constant $M_{3}$ independent of $x, y$. Thus, there exists a constant $M$ such that

$$
\left\|q_{1}(x)\right\|_{C^{0, \alpha+\delta}[b, B]} \leq M\left\|\frac{d}{d x} \frac{1}{x^{\beta-1} f_{2}(x)}\right\|_{C^{0, \alpha}[b, B]} .
$$

In view of (5.2), this is the same as saying that

$$
\left\|q_{1}(x)\right\|_{C^{0, \alpha+\delta}[b, B]} \leq \text { const. }\left\|f_{2}\right\|_{C^{1, \alpha}[b, B]} \cdot
$$


In a similar way, we can show that there exists a constant independent of $f_{2}$ such that

$$
\left\|q_{2}(x)\right\|_{C^{0, \alpha+\delta}[b, B]} \leq \text { const. }\left\|f_{2}-f_{1}\right\|_{C^{1}[b, B]} .
$$

This, together with (5.13) and (5.15), implies that

$$
\left\|p_{3}\right\|_{C^{1, \alpha+\delta}[b, B]} \leq \text { const. }\left\|f_{2}-f_{1}\right\|_{C^{1}[b, B]}\left\|f_{2}\right\|_{C^{1, \alpha}[b, B]} .
$$

Hence, by Lemma 5.2,

$$
\left\|p_{3}\right\|_{C^{1, \alpha+\delta}[b, B]} \leq \text { const. }\left\|F_{2}-F_{1}\right\|_{1, \alpha+\delta, \eta}\left\|f_{2}\right\|_{C^{1, \alpha}[b, B]} .
$$

Combining this with (5.11), (5.12), we arrive at

$\left\|\left(I_{0, w_{2}}^{\delta}-I_{0, w_{1}}^{\delta}\right) \frac{1}{x^{\beta-1} f_{2}(x)}\right\|_{C^{1, \alpha+\delta}[b, B]} \leq$ const. $\left\|F_{2}-F_{1}\right\|_{1, \alpha+\delta, \eta}\left(1+\left\|f_{2}\right\|_{C^{1, \alpha}[b, B]}\right)$.

This, together with (5.10), yields

$$
\left\|\left(I_{0, w_{2}}^{\delta}-I_{0, w_{1}}^{\delta}\right) \frac{1}{x^{\beta-1} f_{2}(x)}\right\|_{1, \alpha+\delta, \eta+1-\beta+\beta \delta} \leq \text { const. }\left\|F_{2}-F_{1}\right\|_{1, \alpha+\delta, \eta}\left(1+\left\|f_{2}\right\|_{C^{1, \alpha}[b, B]}\right) .
$$

Therefore, by (5.9),

$$
\left\|\frac{1}{x^{\beta-1} f_{2}(x)}-\frac{1}{x^{\beta-1} f_{1}(x)}\right\|_{1, \alpha, \eta+1-\beta} \leq \text { const. }\left\|F_{2}-F_{1}\right\|_{1, \alpha+\delta, \eta}\left(1+\left\|f_{2}\right\|_{C^{1, \alpha}[b, B]}\right)
$$

because $I_{0, w_{1}}^{\delta}$ is a homeomorphism of $\mathcal{C}^{1, \alpha}[0, B]_{\eta+1-\beta}$ onto $\mathcal{C}^{1, \alpha+\delta}[0, B]_{\eta+1-\beta+\beta \delta}$. This implies that

$$
\left\|f_{2}-f_{1}\right\|_{C^{1, \alpha}[b, B]} \leq \text { const. }\left\|F_{2}-F_{1}\right\|_{1, \alpha+\delta, \eta}\left(1+\left\|f_{2}\right\|_{C^{1, \alpha}[b, B]}\right) .
$$

Hence

$$
\begin{aligned}
\left\|f_{2}\right\|_{C^{1, \alpha}[b, B]} & \leq\left\|f_{1}\right\|_{C^{1, \alpha}[b, B]}+\left\|f_{2}-f_{1}\right\|_{C^{1, \alpha}[b, B]} \\
& \leq\left\|f_{1}\right\|_{C^{1, \alpha}[b, B]}+\text { const. }\left\|F_{2}-F_{1}\right\|_{1, \alpha+\delta, \eta}\left(1+\left\|f_{2}\right\|_{C^{1, \alpha}[b, B]}\right) .
\end{aligned}
$$

It follows from this estimate that if $\left\|F_{2}-F_{1}\right\|_{1, \alpha+\delta, \eta}$ is sufficiently small then $\left\|f_{2}\right\|_{C^{1, \alpha}[b, B]} \leq \exists M_{1}$. Hence, by (5.17), if $\left\|F_{2}-F_{1}\right\|_{1, \alpha+\delta, \eta}$ is sufficiently small then

$$
\left\|\frac{1}{x^{\beta-1} f_{2}(x)}-\frac{1}{x^{\beta-1} f_{1}(x)}\right\|_{1, \alpha, \eta+1-\beta} \leq M_{2}\left\|F_{2}-F_{1}\right\|_{1, \alpha+\delta, \eta} .
$$

By (5.2) we arrive at $\left\|f_{2}-f_{1}\right\|_{1, \alpha, \eta} \leq M\left\|F_{2}-F_{1}\right\|_{1, \alpha+\delta, \eta}$, provided that $\| F_{2}-$ $F_{1} \|_{1, \alpha+\delta, \eta}$ is sufficiently small. The proof is complete.

We are now in a position to establish the continuity of the transform $\mathcal{K}$.

Proposition 5.4. Under the same assumptions as in Theorem 1.1, the inverse transform $\mathcal{K}^{-1}: \mathcal{M}^{1, \alpha+\delta}(I)_{\eta} \rightarrow \mathcal{M}^{1, \alpha}(I)_{\eta}$ is continuous.

Proof. It suffices to treat the case where $I=[0, B]$. By Lemma 5.3, the inverse of the map $\mathcal{K}_{0}$ in the diagram (2.4) is continuous. Since the vertical arrows $\omega_{\alpha}, \omega_{\alpha+\delta}$ in the diagram are homeomorphisms, the inverse transform $\mathcal{K}^{-1}$ is continuous. 


\section{Bifurcation transform}

It is well-known in a general framework of bifurcation theory (see, e.g., Crandall and Rabinowitz [5], Rabinowitz [24]) that if $f$ is a continuous function on $\mathbb{R}$ with $f(0)>0$ then the $n$-th bifurcating branch of the nonlinear Sturm-Liouville equation

$$
\left\{\begin{array}{l}
u^{\prime \prime}+\lambda u f(u)=0 \quad \text { on }(0,1), \\
u(0)=u(1)=0
\end{array}\right.
$$

bifurcates at the point $\left(\frac{(n \pi)^{2}}{f(0)}, 0\right)$ from the set $\left\{\left(\lambda_{n}, 0\right): \lambda \in \mathbb{R}\right\}$ of the trivial solutions, where $\lambda_{n}:=\frac{(n \pi)^{2}}{f(0)}, n=1,2, \cdots$, are the eigenvalues of the linearized problem

$$
\left\{\begin{array}{l}
u^{\prime \prime}+\lambda u f(0)=0 \quad \text { on }(0,1), \\
u(0)=u(1)=0
\end{array}\right.
$$

of (6.1). Here, by a solution to (6.1), we mean a pair $(\lambda, u)$ in $\mathbb{R} \times C^{2}[0,1]$ satisfying (6.1). Let $E$ be the space of $C^{1}$ functions on $[0,1]$ satisfying the boundary condition in (6.1), let $\mathcal{S}_{n}$ be the set of solutions $(\lambda, u)$ to (6.1) in which $u$ has exactly $n-1$ zeros in $(0,1)$, and let $\overline{\mathcal{S}_{n}}$ be the closure of $\mathcal{S}_{n}$ in $\mathbb{R} \times E$. A maximal (with respect to inclusion), closed connected subset of $\overline{\mathcal{S}_{n}}$ containing $\left(\lambda_{n}, 0\right)$ is referred to as the $n$th bifurcating branch of (6.1). For each $n=1,2, \cdots$, the $n$th bifurcating branch is an unbounded set (see Rabinowitz [24,25]).

We now suppose that $f(u)>0$ on $I$ where $I$ is a bounded, closed interval containing 0 on $\mathbb{R}$ and focus our attention on the first bifurcating branch, in which $u$ is a positive or negative solution of (6.1). Then we have the following:

Lemma 6.1. We suppose that $f$ is a positive continuous function on I. If $(\lambda, u)$ is a solution of (6.1) satisfying $u \neq 0$ on the interval $(0,1)$ then $\lambda>0$ and $u$ attains its maximum value or minimum value at the middle point $\frac{1}{2}$ of the interval $(0,1)$. The value $h \in I \backslash\{0\}$ and $\lambda$ are related via

$$
\int_{0}^{1} \frac{d t}{\left(\int_{t}^{1} s f(h s) d s\right)^{1 / 2}}=\sqrt{\frac{\lambda}{2}} .
$$

Proof. Let $(\lambda, u)$ be a solution of (6.1). Then, by an integration by parts, we have

$$
0=\int_{0}^{1} u(t) u^{\prime \prime}(t)+\lambda u(t)^{2} f(u(t)) d t=-\int_{0}^{1} u^{\prime}(t)^{2} d t+\lambda \int_{0}^{1} u(t)^{2} f(u(t)) d t
$$

Since $f>0$, this implies that $\lambda>0$. By a standard calculation, it follows that $u=u(t)$ satisfies

$$
u^{\prime}(t)^{2}+2 \lambda \int_{0}^{u(t)} r f(r) d r=u^{\prime}(0)^{2}, \quad t \in[0,1] .
$$


This shows that $u^{\prime}(0) \neq 0$ and the value of $u$ at the point $t_{0}$ where $u^{\prime}\left(t_{0}\right)=0$ is uniquely determined by this identity. We denote this value $u\left(t_{0}\right)$ by $h$. Then $u^{\prime}(0)^{2}=2 \lambda \int_{0}^{h} r f(r) d r$, and therefore, by (6.3),

$$
u^{\prime}(t)^{2}=2 \lambda \int_{u(t)}^{h} r f(r) d r .
$$

Therefore, if $u(t)>0$ for $t \in(0,1)$, namely if $h>0$, then

$$
\begin{aligned}
& t_{0}=\int_{0}^{h} \frac{d t}{d u} d u=\frac{1}{\sqrt{2 \lambda}} \int_{0}^{h} \frac{d u}{\sqrt{\int_{u}^{h} r f(r) d r}}, \\
& 1-t_{0}=\int_{h}^{0} \frac{d t}{d u} d t=-\frac{1}{\sqrt{2 \lambda}} \int_{h}^{0} \frac{d u}{\sqrt{\int_{u}^{h} r f(r) d r}} .
\end{aligned}
$$

This shows that $t_{0}=\frac{1}{2}$ and

$$
\int_{0}^{h} \frac{d u}{\sqrt{\int_{u}^{h} r f(r) d r}}=\sqrt{\frac{\lambda}{2}} .
$$

By the substitutions $u=h t, r=h s$, we arrive at (6.2). The case $h<0$ can be treated in a similar manner.

Let $\mathcal{S}_{1}^{I}$ be the set of solutions $(\lambda, u)$ in $(0, \infty) \times E$ to $(6.1)$ in which $u \neq 0$ on $(0,1)$ and $u(t) \in I$ on $[0,1]$. We define a map $\varpi: \mathcal{S}_{1}^{I} \rightarrow(0, \infty) \times I$ by $\varpi:(\lambda, u) \mapsto\left(\lambda, u\left(\frac{1}{2}\right)\right)$. Then $\varpi$ is a continuous map from $\mathcal{S}_{1}^{I}$ onto $\Gamma(f)$, where $\Gamma(f)$ is the set defined in (1.7).

Lemma 6.1 tells us that $\Gamma(f)$ is written as $\Gamma(f)=\{(\lambda(h), h): h \in I \backslash\{0\}\}$, where $\lambda(h)$ is a function on $I$ defined by (1.8). The correspondence $\mathcal{B}: f(u) \mapsto$ $\lambda(h)$ is referred to as the bifurcation transform:

$$
(\mathcal{B} f)(h)=2\left(\int_{0}^{1} \frac{d t}{\sqrt{\int_{t}^{1} s f(h s) d s}}\right)^{2} .
$$

The inverse bifurcation problem we are concerned with consists of three questions:

\section{Problem 6.2.}

1. (Existence) Given a positive function $\lambda$ on $I$, does there exist a positive function $f$ on $I$ such that $\mathcal{B} f=\lambda$ ?

2. (Uniqueness) Is $f$ unique for each $\lambda$ ?

3. (Stability) Does $f$ depend on $\lambda$ continuously ? 
By (6.5), the bifurcation transform $\mathcal{B}$ is written as $\mathcal{B} f=2(\mathcal{K} f)^{2}$ in terms of the transform $\mathcal{K}$ with $\delta=\frac{1}{2}, \beta=2$ in (1.1). But, as is easily verified, the transform $g \mapsto 2 g^{2}$ is a homeomorphism of $\mathcal{M}^{1, \alpha+\frac{1}{2}}(I)_{\eta}$ onto itself when $\eta>0$. Accordingly, as an immediate consequence of Theorem 1.1, we obtain the following answer (Theorem 1.2): the bifurcation transform $\mathcal{B}$ is a homeomorphism of $\mathcal{M}^{1, \alpha}(I)_{\eta}$ onto $\mathcal{M}^{1, \alpha+\frac{1}{2}}(I)_{\eta}$, provided that $0<\eta \leq \alpha<\frac{1}{2}$.

Example 6.3. Let $I=[a, b]$ with $-1<a \leq 0 \leq b<\infty$, and, for a given function $\lambda(h):=\left(\sqrt{h+1}+\frac{\pi}{2}-1\right)^{2}$, consider $\mathcal{B} f=\lambda$. Obviously, $\lambda(h)$ is a positive function on $I$ belonging to $C^{2}(I)$. Hence, for each $\alpha, \eta$ such that $0<\eta \leq$ $\alpha<\frac{1}{2}$, the function $\lambda(h)$ belongs to $\mathcal{M}^{1, \alpha+\frac{1}{2}}(I)_{\eta}$. By Theorem 1.2, there exists a unique function $f=f(u)$ in $\mathcal{M}^{1, \alpha}(I)_{\eta}$ such that $\mathcal{B} f=\lambda$. The function $f(u)$ is determined as

$$
f(u)=\frac{8}{u+1+\sqrt{u+1}},
$$

because, for the function $f$,

$$
\begin{aligned}
(\mathcal{B} f)(h) & =\frac{1}{4}\left(\int_{0}^{1} \frac{h}{\sqrt{(\sqrt{h+1}-1)^{2}-(\sqrt{h t+1}-1)^{2}}} d t\right)^{2} \\
& =\left(\int_{0}^{\frac{\pi}{2}}(1+(\sqrt{h+1}-1) \sin \varphi) d \varphi\right)^{2}=\left(\sqrt{h+1}+\frac{\pi}{2}-1\right)^{2},
\end{aligned}
$$

where we have used the substitution $\sqrt{h t+1}-1=(\sqrt{h+1}-1) \sin \varphi$. Thus we conclude that

$$
\mathcal{B}^{-1}\left(\sqrt{h+1}+\frac{\pi}{2}-1\right)^{2}(u)=\frac{8}{u+1+\sqrt{u+1}}, \quad 0 \leq u \leq b .
$$

The solution $u=u(t ; h)$ on the point $(\lambda(h), h)$ (see Figure 6.1) is given as the inverse function of

$$
t(u)=\frac{1}{2 \sqrt{\lambda(h)}}\left\{A-\sqrt{A^{2}-(\sqrt{u+1}-1)^{2}}+\sin ^{-1} \frac{\sqrt{u+1}-1}{A}\right\},
$$

where $A=\sqrt{h+1}-1$. The set $S_{1}^{I}$ of solutions $(\lambda, u)$ to (6.1) in which $u(t) \neq 0$ on $(0,1)$ and $u(t) \in I$ is determined as $\{(\lambda(h), u(t ; h)): h>0\}$. It should be noted that, since $f$ determines all the solutions such that $u(t) \in I, \lambda(h)$ determines all the solutions in $S_{1}^{I}$.

Though the function $f=\mathcal{B}^{-1} \lambda$ belongs to $\mathcal{M}^{1, \alpha}[a, b]_{\eta}$ for any $b>0$, it does not belong to $\mathcal{M}^{1, \alpha}[-1, b]_{\eta}$ since it blows up at $h=-1$. The reason is that the function $\lambda$ does not belong to $\mathcal{M}^{1, \alpha+\frac{1}{2}}[-1, b]_{\eta}$ (the derivative $\lambda^{\prime}(h)$ is 


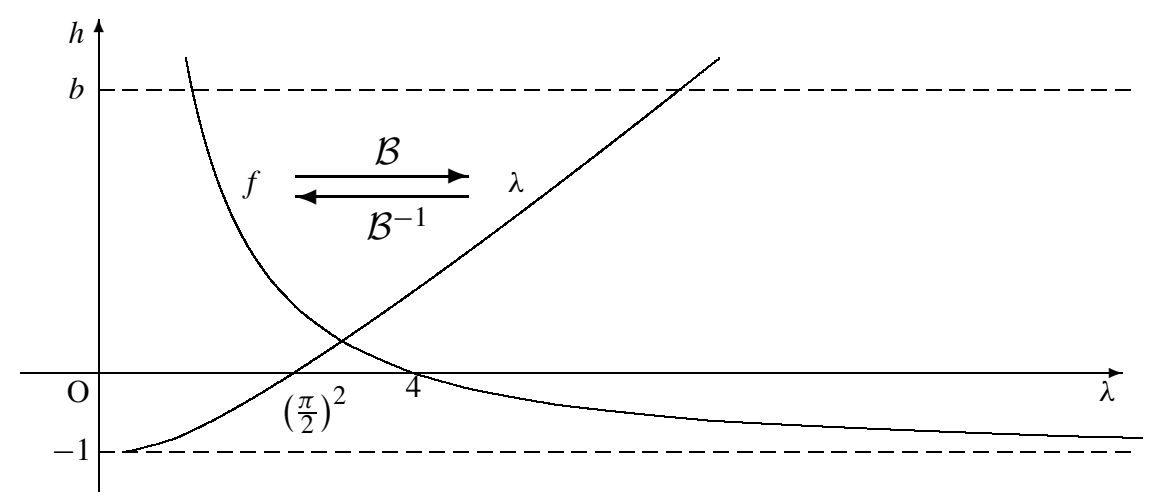

Figure 6.1. Case where $\lambda(h)=\left(\sqrt{h+1}+\frac{\pi}{2}-1\right)^{2}$.

not continuous at $h=-1$ ). This situation is the same as that in the case $\frac{\delta-1}{2-\delta}<$ $\mu<0$ of Example 4.5; unlike the conclusion in Theorem 1.2, it does not hold in general that the bifurcation transform $\mathcal{B}$ gives a homeomorphism of $\mathcal{M}^{0, \alpha}(I)_{\eta}$ onto $\mathcal{M}^{0, \alpha+\frac{1}{2}}(I)_{\eta}$.

\section{References}

[1] B. ALFAWICKA, Inverse problems connected with periods of oscillations described by $\ddot{x}+$ $g(x)=0$, Ann. Polon. Math. 44 (1984), 297-308.

[2] B. ALFAWICKA, Inverse problem connected with half-period function analytic at the origin, Bull. Pol. Acad. Sci. Math. 32 (1984), 267-274.

[3] A. Cima, A. GASUll and F. Mañosas, Period function for a class of Hamiltonian systems, J. Differential Equations 168 (2000), 180-199.

[4] A. CimA, F. MAÑOSAS and J. VILLADELPRAT, Isochronicity for several classes of Hamiltonian systems, J. Differential Equations 157 (1999), 373-413.

[5] M. G. CRANDALL and P. H. RABINOWitz, Bifurcation from simple eigenvalues, J. Funct. Anal. 8 (1971), 321-340.

[6] A. DENISOv, Inverse problems for nonlinear differential equations, Dokl. Akad. Nauk. 307 (1989), 1040-1042.

[7] A. M. DENISOV and A. LoRENZI, Identification of nonlinear terms in boundary value problems related to ordinary differential equations, Differential Integral Equations 5 (1992), 567-579.

[8] A. M. DENISOV and A. LoRENZI, Recovering nonlinear terms with a priori unknown domains of definition in second order nonlinear differential equations, J. Inverse Ill-Posed Probl. 11 (2003), 137-159.

[9] R. GoRenflo and S. Vessella, "Abel Integral Equations", Lecture Notes in Mathematics, Vol. 1461, Springer, Berlin, 1991.

[10] M. HENRARD and F. ZANOLIN, Bifurcation from a periodic orbit in perturbed planar Hamiltonian systems, J. Math. Anal. Appl. 277 (2003), 79-103.

[11] K. IWASAKI and Y. KAMIMURA, An inverse bifurcation problem and an integral equation of the Abel type, Inverse Problems 13 (1997), 1015-1031. 
[12] K. IWASAKI and Y. KAMIMURA, Convolution calculus for a class of singular Volterra integral equations, J. Integral Equations Appl. 11 (1999), 461-499.

[13] K. IWASAKI and Y. KAMIMURA, Inverse bifurcation problem, singular Wiener-Hopf equations, and mathematical models in ecology, J. Math. Biol. 43 (2001), 101-143.

[14] Y. Kamimura, An inverse problem in bifurcation theory, J. Differential Equations 106 (1993), 10-26.

[15] Y. KAMIMURA, An inverse problem in bifurcation theory, II, J. Math. Soc. Japan 46 (1994), 89-110.

[16] Y. KAMIMURA, An inverse problem of determining a nonlinear term in an ordinary differential equation, Differential Integral Equations 11 (1998), 341-359.

[17] Y. Kamimura, Conductivity identification in the heat equation by the heat flux, J. Math. Anal. Appl. 235 (1999), 192-216.

[18] Y. KAMIMURA, Global existence of a restoring force realizing a prescribed half-period, J. Differential Equations 248 (2010), 2562-2584.

[19] J. B. KelLER, Inverse problems, Amer. Math. Monthly 83 (1976), 107-118.

[20] L. D. Landau and E. M. Lifshitz, "Mechanics", 3rd ed., Course of Mathematical Physics, Vol. 1, Pergamon Press, Oxford, 1976.

[21] A. LORENZI, An inverse spectral problem for a nonlinear ordinary differential equation, Applicable Analysis 46 (1992), 129-143.

[22] F. MAÑOSAS and P. J. TORRES, Two inverse problems for analytic potential systems, J. Differential Equations 245 (2008), 3664-3673.

[23] Z. OPIAL, Sur les périodes des solutions de l'équation differentielle $x^{\prime \prime}+g(x)=0$, Ann. Polon. Math. 10 (1961), 49-72.

[24] P. H. RABINOWITZ, Nonlinear Sturm-Liouville problem for second order ordinary differential equations, Comm. Pure Appl. Math. 23 (1970), 939-961.

[25] P. H. RABINOWITZ, A global theorem for nonlinear eigenvalue problems and applications, In: "Contributions to Nonlinear Functional Analysis", E. H. Zarantonello (ed.), Academic Press, New York-London, 1971, 11-36.

[26] S. G. SAmKo, A. A. KilbaS and O. I. Marichev, "Fractional Integrals and Derivatives", Gordon and Breach, Switzerland, 1993.

[27] R. SchAAF, "Global Solution Branches of Two Point Boundary Value Problems", Lecture Notes in Mathematics, Vol. 1458, Springer, Berlin, 1990.

[28] J. T. Schwartz, "Nonlinear Functional Analysis", Gorgon and Breach, New York, 1969.

[29] T. SHIBATA $L^{q}$-inverse spectral problems for semilinear Sturm-Liouville problems, Nonlinear Anal. 69 (2008), 3601-3609.

[30] M. URABE, The potential force yielding a periodic motion whose period is an arbitrary continuous function of the amplitude of the velocity, Arch. Ration. Mech. Anal. 11 (1962), $27-33$.

[31] M. URABE, Relation between periods and amplitudes of periodic solutions of $\ddot{x}+g(x)=0$, Funkc. Ekvacioj 6 (1964), 63-88.

[32] M. URabe, "Nonlinear Autonomous Oscillations", Academic Press, New York, 1967.

[33] P. ZHIDKOV, On an inverse eigenvalue problem for a semilinear Sturm-Liouville operator, Nonlinear Anal. 68 (2008), 639-644.

Department of Ocean Sciences

Tokyo University of Marine Science and Technology

4-5-7 Konan, Minato-ku

Tokyo 108-8477, Japan

kamimura@kaiyodai.ac.jp 